\title{
Harvesting electricity by harnessing nature: bioelectricity, triboelectricity, and method of storage
}

\author{
Chandu V.V. Muralee Gopi, Tien Khee Ng, and Boon S.Ooi* \\ Photonics Laboratory, King Abdullah University of Science and Technology (KAUST), \\ Thuwal, 23955-6900, Saudi Arabia \\ *Corresponding author: boon.ooi@kaust.edu.sa
}

\begin{abstract}
In nature, bioelectricity refers to electrical potentials and currents produced by or used within living cells, tissues, and organisms for electrolocation, predation, or protection. Electric eels can generate huge amounts of power using electric organs that are arranged in stacks of electrocytes. One critical, recent issue for electronic gadgets is that energy storage systems are incapable of offering enough energy for uninterrupted, long-running processes. This results in frequent recharging or inconvenient energy storage unit replacement. To address this challenge, inspired by bioelectricity phenomena, unique triboelectric nanogenerator (TENG) technology has been proposed to convert small quantities of mechanical energy into electricity without an external power supply. Several advances have been made regarding TENG-based self-charging energy storage devices. To fulfil the sustainable operation requirements of next-generation electronic devices, extensive work has been performed to integrate energy-generating TENGs with energy storing supercapacitor devices to form selfcharging power systems (SCPSs). This tutorial article focuses on recent advances and various SCPS structural designs. In addition, various power management circuits that can be integrated with TENG devices and supercapacitors are reviewed. Finally, challenges and perspectives for future SCPS progress are discussed.
\end{abstract}

Keywords Bioelectricity; electric eel; mechanical energy; triboelectric nanogenerators; supercapacitors; self-charging power systems;

$\begin{array}{llr}1 & \text { Introduction } & 1 \\ 2 & \text { Triboelectric Nanogenerator (TENG) } & 3 \\ 3 & \text { Supercapacitor } & 8 \\ 4 & \text { Bio-Inspired SCPS (Integration of TENG with Supercapacitor) } & 12 \\ 5 & \text { Power Management } & 20 \\ 6 & \text { Summary and Perspective } & 22 \\ & \text { Acknowledgements } & \\ & \text { References }\end{array}$

\section{Introduction}

The extensive worldwide progress in electronic technology follows the general paths of portability, miniaturization, and functionality. With rapid growth of the economy and society, the internet of things (IoT) and electronic gadgets are performing increasingly crucial roles in our daily lives. Electronic devices increasingly operate independently and even 
without physical contact. This is known as the IoT. The IoT requires widely distributed sensors for various applications and the number of such units can vary from billions to trillions. In addition, electronic product development is trending towards small sizes, intelligence, and multifunctional capabilities. This increases energy consumption and power supply requirements [1]. The most promising power supply technologies use electrochemical energy storage devices such as supercapacitors (SCs) and batteries, all of which have limited lifespans. These energy storage devices require exterior power sources for frequent recharging or replacement, which can cause substantial inconvenience and high operating costs [2]. One approach to solving this issue is to improve supercapacitor and battery performance. Substantial research has been devoted to this objective [3]. Development of highly efficient self-charging power systems (SCPS) that properly combine energyharvesting and energy storing units is an alternative strategy. Energy is harvested from the ambient environment and stored to provide a sustainable power supply [4].

Several important forms of energy can be extracted from our environment, such as thermal, solar, biochemical, and mechanical energy. Among them, mechanical energy is widely distributed and nearly independent of the weather and the working environment. Fan et al. [5] recently developed triboelectric nanogenerators (TENGs) that can efficiently convert ambient mechanical energy (e.g., wind, wave, or droplet energy) into electricity via the triboelectric effect and electrostatic induction [6]. Nanogenerators provide several advantages including simplicity, cost effectiveness, size, ease of fabrication, convenience, and environmental friendliness. Thus, they might be integrated with an energy storage unit (SC) that they recharge. Therefore, it is important to investigate SCPSs that can efficiently integrate energy harvesting (TENG) and energy storage units into a single device for sustainable, maintenance-free operation (Figure 1).

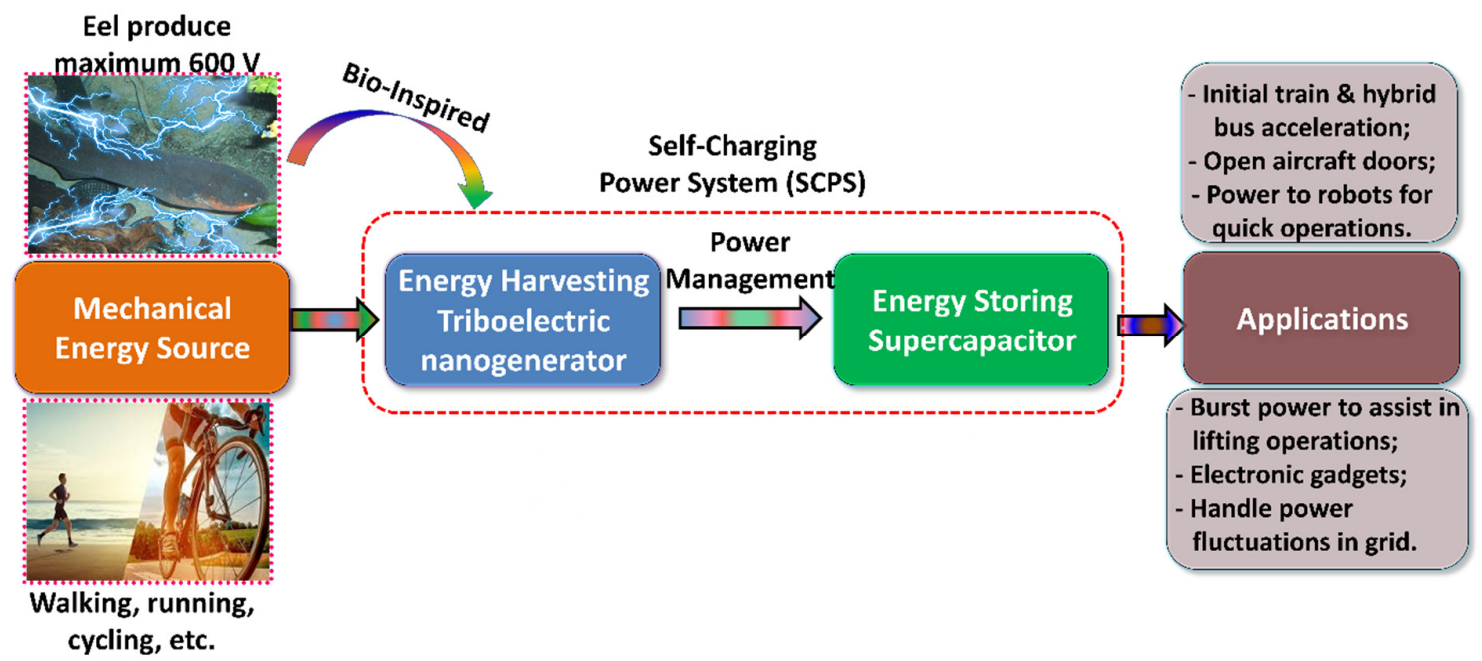

Figure 1. Schematic illustration of bioelectricity inspired self-charging power system (SCPS).

In nature, electric rays and electric eels (Electrophorus electricus) produce electricity using an electric organ (EO) to immobilize prey and defend themselves from possible threats 
[7]. In electric eels, the EO is an arrangement of cells called electrocytes that can provide a maximum voltage of $600 \mathrm{~V}$ (Figure 1) [8]. Inspired by bioelectric production, SCPS research and development is of interest to researchers [9]. Therefore, this tutorial discusses the latest SCPS developments regarding integration of energy-harvesting TENG units with energystoring supercapacitors. First, a brief discussion of TENG and supercapacitor technologies is provided. Next, several SCPS fabrication strategies and potential SCPS application areas are presented and discussed in detail. Finally, some challenges and perspectives for future SCPS progress are reviewed. A schematic of the current review of self-charging power systems is shown in Figure 2.

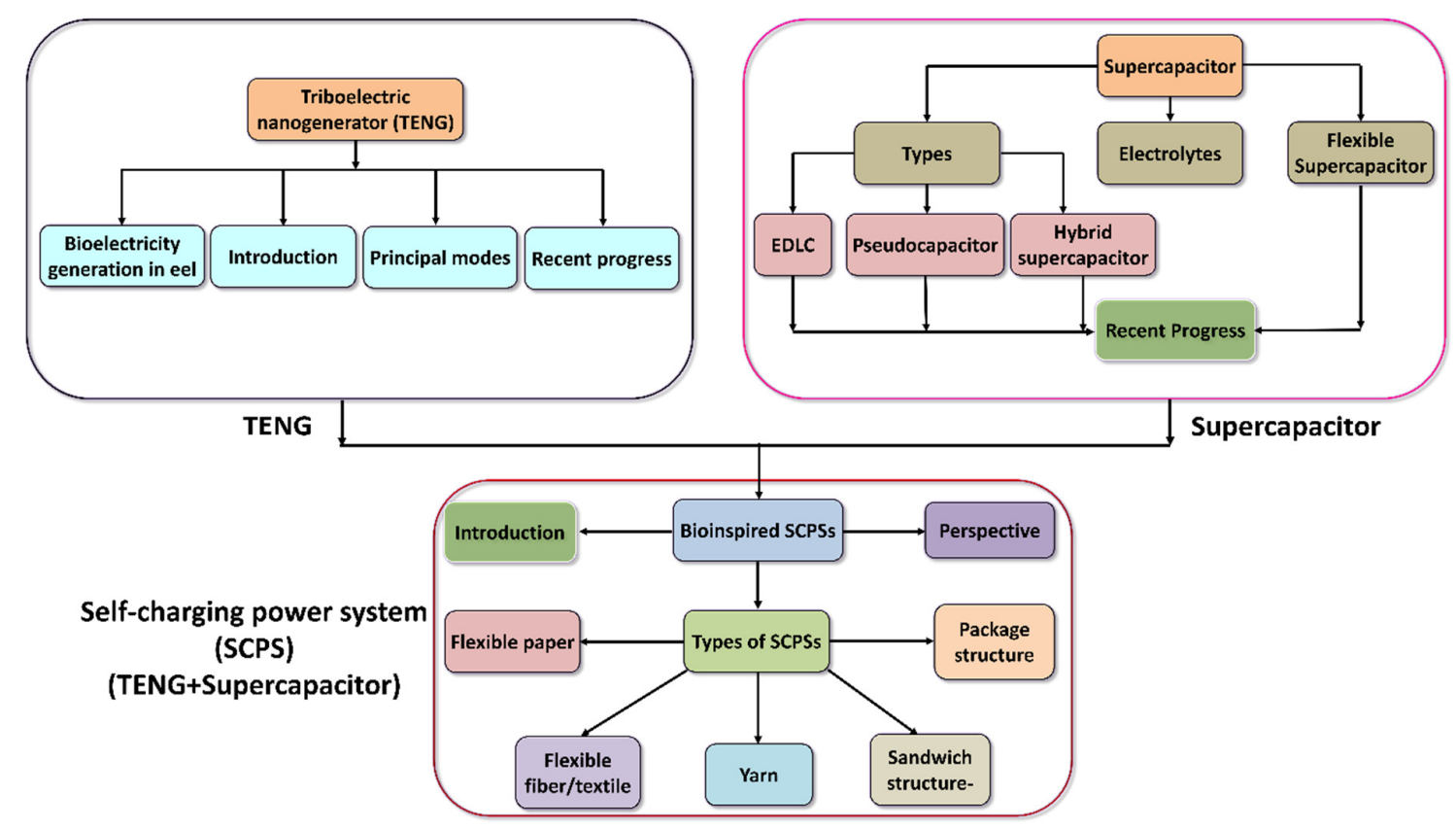

Figure 2. Schematic of the current review self-charging power system content.

\section{Triboelectric Nanogenerator}

\subsection{Bioelectricity Generation in Electric Eel}

Bioelectricity relies on the fact that all animal cells (such as those in electric rays, electric eels, elephant-nose fish, geckos, and Guiana dolphins) possess electrical properties since the potentials inside and outside of cell membranes are different [7,10,11]. Semipermeable cell membranes act as bridge barriers to ions. The fluid ions in the cell membrane are different from those in the cytosol (cell cytoplasm fluid). Chloride and potassium ions can diffuse across the membrane easily, but there is no facility for diffusion of sodium ions. A temporary variation in the cell membrane permeability occurs upon chemical or electrochemical stimulation of a neuron. This causes variation in the ion concentration, which generates electrical charge variation that leads to the necessary potential.

The electric eel (Electrophorus electricus) produces a large electric current via a highly sophisticated nervous system that is capable of synchronizing the behaviours of 
electricity-producing, disc-shaped cells (electrocytes). These are stacked within a specialized electric organ [9]. The eel utilizes transient current peaks to protect itself and to locate and incapacitate prey. When the EO is in its resting state, the electrocytes have higher and lower concentrations of sodium $\left(\mathrm{Na}^{+}\right)$and potassium $\left(\mathrm{K}^{+}\right)$ions, respectively, than the volume outside of the cell membrane. When the EO is activated, cell membrane ion channels form because of a neurotransmitter that permits ions to pass across the cell membrane using the polarized $\mathrm{Na}^{+}$and $\mathrm{K}^{+}$concentration gradients. The transmembrane voltage of an individual electrocyte can reach approximately $150 \mathrm{mV}$. By stacking thousands of electrocytes in series, a large electric eel can produce a maximum voltage of approximately $600 \mathrm{~V}$ [12]. Using bioinspired self-powered energy generation systems, researchers have recently focused on fabrication of SCPSs by integrating TENG units with supercapacitors. This has applications in the IOT and electronic devices [9]. TENG, supercapacitor, and SCPS fabrication strategies are illustrated in subsequent sections.

\subsection{Introduction to TENG}

(a)

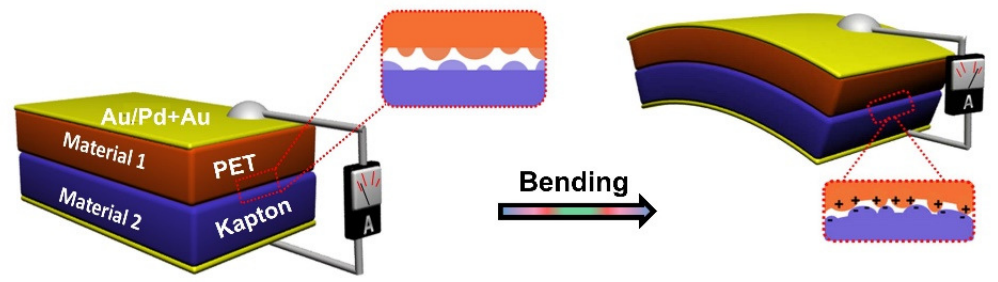

(b)

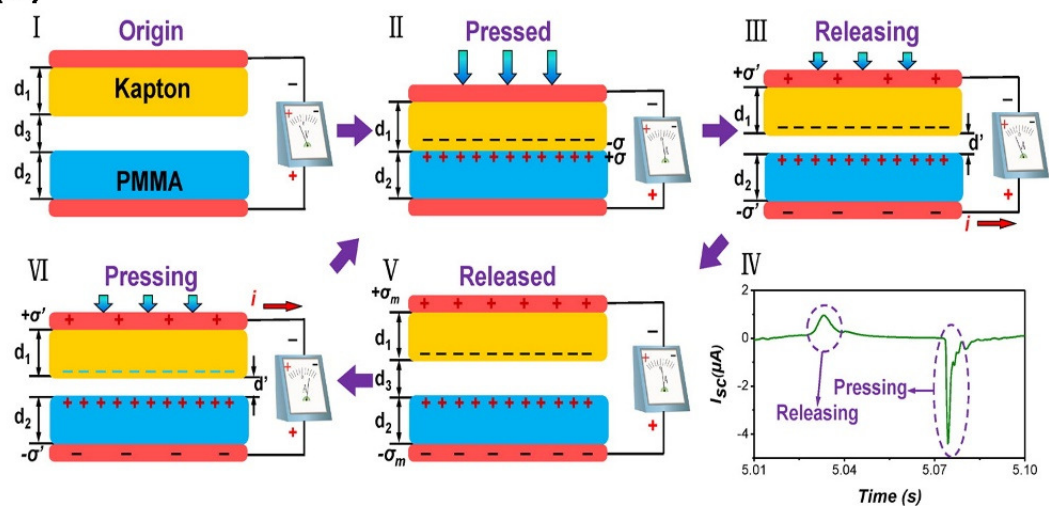

Figure 3. (a) The structure and photographs of the first TENG. Source: Fan et al. [5] Reprinted with permission of Elsevier. (b) Working principle of the TENG. Source: Zhu et al. [15]. Reprinted with permission ACS publications.

Because of increasingly severe energy deficits, ecological issues, and an increasing need for electronics, it is important to examine new clean, portable energy resources. Extensive research has been performed with the goal of harvesting energy from ambient environmental sources (mechanical energy, wind power, solar energy, etc.) as a possible solution for the energy crisis. Because they scavenge energy from various environmental sources, development of new energy-enhancing equipment is essential to addressing future functional electronics power source issues. In this section, we discuss bio-inspired TENGs and recent related advances. In 2012, Wang's group developed a new TENG energy 
harvesting and conversion device that efficiently converts various mechanical energies into electricity via electrostatic induction and triboelectrification [5]. TENGs offer other benefits such as high specific power, low operating frequencies, cost effectiveness, and simplicity of fabrication. Thus, there is considerable interest in TENG-based energy harvesting [13]. Moreover, TENGs have been employed to extract energy from body movement, vibration, wind, and waves. Various TENG devices that work via various modes have been explored as wearable electronic devices [14].

The fundamental structure of a basic TENG is depicted schematically in Figure 3a [5]. It is composed of polyester (PET) and Kapton films with back electrodes attached. The device can produce AC output when contact between the two electrodes is altered, causing it to undergo cyclic compression or bending motions. The detailed working mechanism of the TENG is shown in Figure 3b [15]. If two different material surfaces come into physical contact, the two insulating polymeric materials touch and rub each other such that oppositely signed triboelectric charges are produced and distributed across the two contacting surfaces. Here, the Poly(methyl methacrylate) (PMMA) and Kapton films are positively and negatively charged. They establish an interfacial dipole layer, which is also referred to as a triboelectric potential layer. Therefore, a potential difference forms when the films are kept apart via mechanical movement and charges can transport between the two electrodes via an external circuit.

Recently, Gallant et al. demonstrated the basic theory of TENGs, which can be traced back to Maxwell's displacement current [16]. The TENG output current is proportional to Maxwell's displacement current $\left(\mathrm{J}_{\mathrm{D}}\right)$ according to:

$$
J_{D}=\frac{\partial \mathrm{D}}{\partial \mathrm{t}}=\varepsilon_{o} \frac{\partial \mathrm{E}}{\partial \mathrm{t}}+\frac{\partial \mathrm{P}_{s}}{\partial \mathrm{t}}
$$

where D is the electric displacement vector, $\mathrm{E}$ is the electric field, $\mathrm{P}_{\mathrm{S}}$ is the polarization field, and $\varepsilon_{\mathrm{o}}$ is the permittivity in vacuum. In Maxwell's displacement current, the first term relates to the time-varying electric field and has its origin in electromagnetic waves, whereas the second term denotes the current contribution from surface polarization. Thus, nanogenerators arise. 


\subsection{Principal TENG Modes}

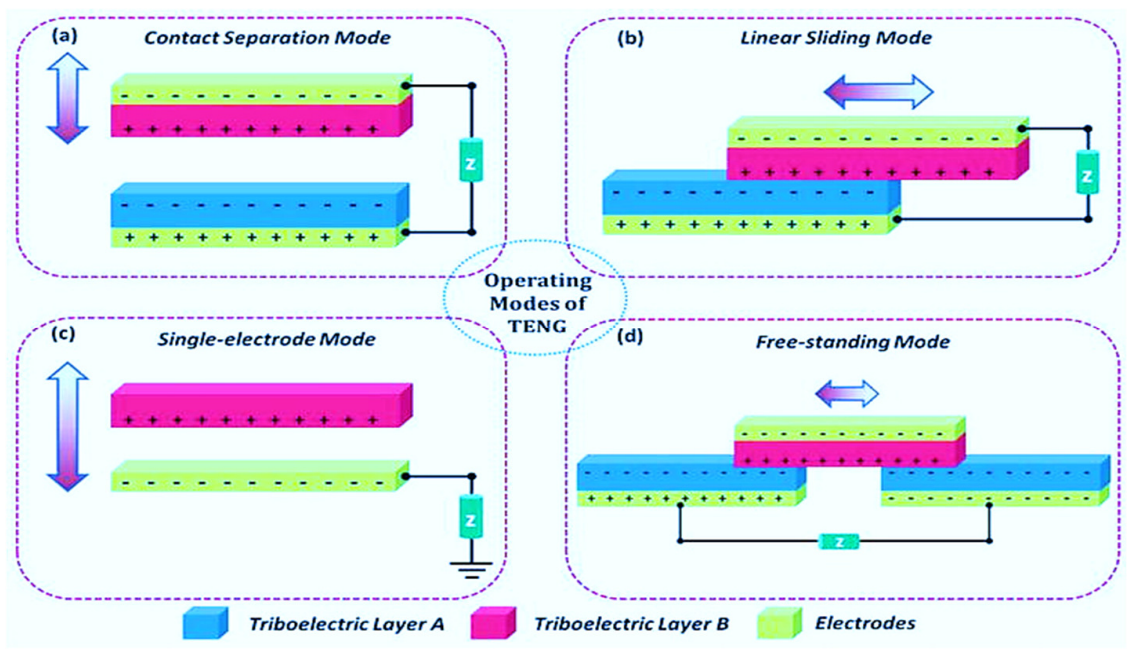

Fig. 4. The four working modes of the TENG. (a) The vertical CS mode. (b) The LS mode. (c) The SE mode. (d) The FT mode [17].

Based on the electrode configuration and directional variation of polarization, four types of TENG operating modes are generally expected and are depicted in Fig. 4 [17]. These are the vertical contact separation (CS), lateral sliding (LS), single-electrode (SE), and freestanding triboelectric-layer (FT) modes. These modes utilize two different triboelectric materials with proper insulation and proper electrode connections between each layer. The combination may be either dielectric-dielectric or metal-dielectric arrangements. The vertical CS mode utilizes relative displacement vertical to the interface. In detail, the two terminal contact surfaces have opposite electrical polarities and a small air gap exists between them (as shown in Fig. 4a). When the two terminals are come into contact via mechanical force from water waves, a positive triboelectric charge is induced on one surface and a negative charge is produced on other side. Upon removal of the external force, the terminals are separated. This generates opposite charges on the surfaces and a current is produced in the external circuit. Later, these two terminals come back into contact and the process repeats. This model has a noteworthy benefit with its simple strategy, easy fabrication, and costeffective. This CS mode is first developed and demonstrated for powering low-power electronics.

Similarly, the lateral sliding (LS) mode utilizes the relative motion parallel to the interface in the form of relative sliding of the two contacting surfaces (Fig. 4b). The LS mode fabrication is similar to the CS mode, but the displacement is in sideward. The LS mode can produce more charge density with a highly effective charge generation than that of CS mode due to its high contact area. In contrast, the single-electrode (SE) mode can generate energy from free-moving objects (such as a hand typing, walking, and other transportation) (Fig. 4c). The SE mode overcomes the application limitation due to the contact wire obstructing at both sides in the CS mode and LS mode TENG devices. The free-standing (FT) mode has one electrode move freely between the two electrodes or triboelectric layers. The freestanding triboelectric-layer (FT) mode generates power via electrostatic induction between a pair of 
electrodes (Fig. 4d). The theoretical models of the four modes were comprehensively examined and studied in previous literature, and thus are not covered in detail here [18].

\subsection{Progress in TENG-based Design Configurations that Use Mechanical Energy}

Many researchers have tried to develop new structures for TENG devices that harvest environmental mechanical energy such as human movements, wind, and tidal energy [19-27]. Using the triboelectric and electrostatic effects, two distinct electronegative TENG materials are contacted and separated, thus allowing excited electrons to pass through them. The TENGs' high power density, flexible architectural design, broad material selection, and reliable manufacturing are the universal criteria for mechanical energy harvesting [28]. For possible applications and improved output, development of sustainable and cost-effective TENGs with various structures and functions is critical to adapting to various environmental parameters.

In nature, electric eel skin has both the benefits of softness and the ability to generate power when the animal is attacked by a predator. Electric eel skin power mimics not only provide skin-specific features and physical compatibility, but also a self-contained interactive interface. This competes with resistive and capacitive touch sensors that need strong power supplies [29]. Scientists have adopted electric eel structures to produce high-output-voltage, flexible, fiber-based capacitors [30]. Tenderness, body and shape compliance, and the ability to generate electricity are important unique characteristics of electric eel skin. To duplicate these properties, Lai et al. demonstrated a skin-like triboelectric nanogenerator that was mechanically durable and resilient [31]. Highly stretchable, durable sliver nanowires (Ag NWs) were embedded in silicone rubber. There was no significant decrease in the $\mathrm{J}_{\mathrm{SC}}$ when the charging resistance was below $100 \mathrm{M} \Omega$. When the resistance was increased to $200 \mathrm{M} \Omega$, a maximum power density of $0.5 \mathrm{~W} \mathrm{~m}^{-2}$ could be attained. Also, the device exhibited no $J_{\mathrm{sc}}$ degradation even over 3600 cycles. Finally, the highly efficient skin-like triboelectric nanogenerator powered serially connected LEDs via gentle touching.

Early researchers sought to understand the bioelectrical process primarily by identifying acetylcholine receptors and discussing the development of electric organs (EOs) in the electric eel [32]. However, single-electrolyte EOs can only generate small voltages. They can produce large voltages only when stacked sequentially. For single triboelectric nanogenerators (TENGs), two fundamental operating modes and various materials can be developed to improve output power. In addition, some large-scale methods have been developed to improve TENG output performance. Although various types of TENGs have been developed, most provide high voltages but low currents. This is a substantial problem in practical use. Increasing the energy density is important to development of TENGs. However, combining multiple TENGs into a single controllable energy system is quite rare. Since traditional, large-scale syntheses lacked complex fabrication methods, easy, efficient approaches to combining TENGs are needed but unavailable. 
Using the concepts of electric discharge and electric organ microstructures, Jie et al. sought to improve output currents by developing a structural bionic layout for multi-layered, systematically structured TENGs [33]. The structural bionic design comprises five archshaped TENGs that are integrated such that they all contact simultaneously when a force is applied. The systematically designed TENGs are inter-connected in parallel. An external pressure is applied using an industrial linear mechanical motor (or via hand tapping) to measure the TENG output power. When the number of TENG units increases, the shortcircuit current increases but the open circuit-voltage remains constant. This can be ascribed to the parallel-connected layout. The output voltage improves upon increasing the external load resistance from $10^{5} \Omega$ to $10^{9} \Omega$, while the current exhibits the opposite trend. The maximum output power is $5 \times 10^{6} \Omega$. Matching the external load resistance is essential to maximizing the output power from a system of TENGs. Multiple TENGs were connected in a design intended to provide bionic power to several LEDs.

TENG triboelectric material is generally separated by electronegativity and most positive materials are metals [34]. Moreover, TENG stability is affected severely when the metal-based materials undergo oxidation or corrosion in unfavourable environments [35]. Hence, one current problem in TENG manufacturing is development of high-performance, flexible positive materials. For example, Wang et al. fabricated an all-polymer TENG using polypyrrole and PTFE as the positive and negative materials, respectively, and achieved a power density of $1.44 \mathrm{~W} \mathrm{~m}^{-2}$. However, this is much lower than the power densities of metalbased TENGs [35]. As a result, development of all-polymer-based TENGs with high triboelectric efficiencies is highly desired. To address this, Zheng et al. fabricated polymer porous aerogel film-based triboelectric nanogenerators (A-NGs), which is made of a pair of highly porous polymer films and achieved a maximum power density of $2.33 \mathrm{~W} \mathrm{~m}^{-2}$ [36]. Later, Mi et al. reported a high power density of $3.4 \mathrm{~W} \mathrm{~m}^{-2}$ using a cellulose nanofibril $(\mathrm{CNF}) /$ rabbit fur composite aerogel-based TENG that exhibited improved sensitivity during sensing of mechanical motion, vibration, and small forces [37]. Recently, Zhang et al. developed a cost-effective, highly optically transparent, light-weight gear-like TENG with excellent mechanical features based on biodegradable cellulose nanofibrils (CNFs) for energy harvesting [38]. Compared to regular cellulose fiber, cellulose nanofibrils are rich in hydroxyl groups that significantly enhance their chemical conversion [39].

Designing cost-effective, reliable TENGs with large areal capacitances using easy, consistent, and eco-friendly techniques remains a challenge. To address this challenge, Liu et al. fabricated a large-scale TENG $(4 \mathrm{~cm} \times 6 \mathrm{~cm})$ by spray-coating a transparent silk-fibroin triboelectric layer on a PET/ITO substrate. The resulting device achieved a maximum power density of $68.0 \mathrm{~mW} / \mathrm{m}^{2}$ [40]. More recently, using the melt blowing method, Zheng et al. developed a cost-effective, highly stable, large-area $\left(100 \mathrm{~mm}^{2} \times 100 \mathrm{~mm}^{2}\right)$ fabric-based TENG (FB-TENG) composed of polypropylene non-woven fabrics, commercial polyamide 66 fabrics, and conductive fabrics [41]. The resulting FB-TENG achieved a maximum output power density of $901.7 \mathrm{~mW} / \mathrm{m}^{2}$ and was successfully applied to real-time applications including tracking of self-powered motion, tactile sensing, and monitoring of a remote wireless training system. In 2020, Wang et al. fabricated large-scale $\left(6 \mathrm{~cm}^{2} \times 8 \mathrm{~cm}^{2}\right)$ multifunctional, robust liquid-metal/polymer core/shell fibre-based textile-TENGs (LCF- 
based t-TENGs) via a facile and universal fabrication route [42]. The LCF structures that were the main components in the t-TENGs were prepared by pumping liquid metal into uniform, ultrafine polymer hollow fibers. The LCF-based t-TENGs achieved a maximum power density of $30.4 \mathrm{~mW} / \mathrm{m}^{2}$ and enhanced performance in self-powered sensors used in smart home control applications. However, it is necessary to design and develop more effective, robust, durable, environmentally sustainable, application-compatible materials and also device architectures are essential.

The irregular TENG alternating-current (AC) output cannot be used to operate most electronic devices directly [41,42]. To address this issue, an energy storage device, such as a supercapacitor, must be used to store the harvested TENG energy and deliver direct-current (DC) output to electronic devices. Hence, the design and development of high-performance supercapacitors is highly desirable to effectively extract the energy harvesting TENG output. Supercapacitor technologies are reviewed in the subsequent section.

\section{Supercapacitor}

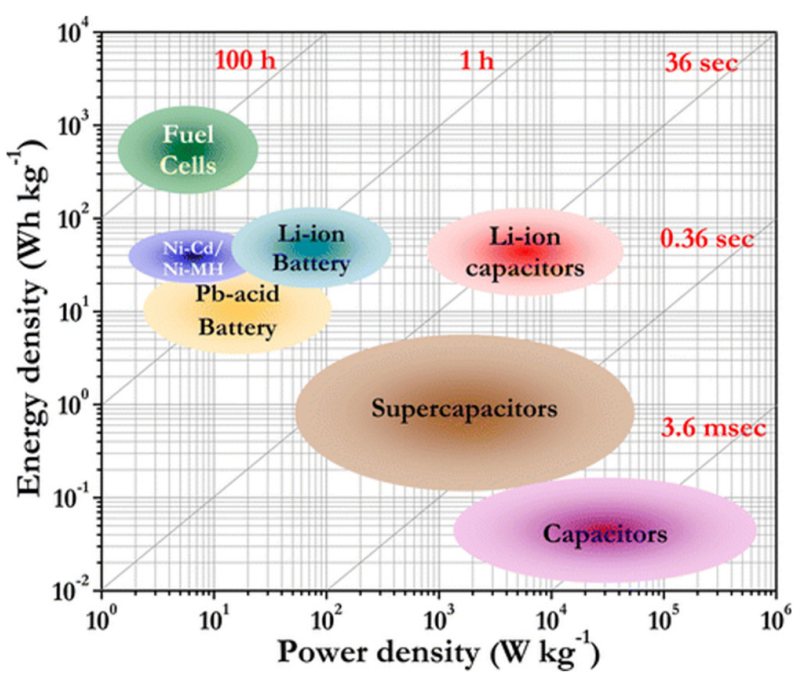

Figure 5. Ragone plot of performance ranges of different energy storage devices. Source: Aravindan et al. [45]. Reprinted with permission of ACS publications.

In recent years, electrochemical energy storage systems including lithium ion batteries (LIBs), fuel cells, and supercapacitors have received substantial attention due to declines in fossil fuels, environmental degradation, and the energy crisis [43]. Supercapacitors have garnered considerable interest for use in various future energy storage devices, as they offer fast charge and discharge rates, high specific power, and excellent cycle life [44]. However, supercapacitors deliver lower specific energies than LIBs $\left(\sim 200 \mathrm{Wh} \mathrm{kg}^{-1}\right)$ and fuel cells $(\sim 350$

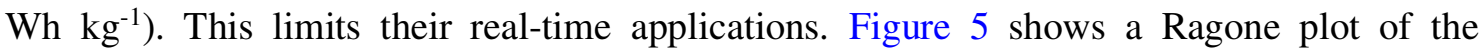
performance ranges of various energy storage devices [45]. Based on the equation $\mathrm{E}=0.5 \mathrm{CV}^{2}$, the specific energy of a supercapacitor can be improved by either enlarging the potential window or achieving a high specific capacitance. Supercapacitor performance relies heavily on the electroactive material that the component contains. Hence, it is valuable to fabricate high-performance electroactive materials to achieve high capacitances and high specific 
energies. In subsequent sections, we discuss supercapacitors and recent advances in flexible supercapacitors.

\subsection{Types of Supercapacitor}

Supercapacitors are categorized into three types based on their charge storage phenomena and electroactive materials: electrochemical double layer capacitors (EDLCs), pseudocapacitors, and hybrid supercapacitors [46]. In EDLCs, energy is stored electrostatically at the electrode/electrolyte interface without Faradaic reactions, resulting in fast charge-discharge rates and excellent cycling performance (Figure 6a) [47]. EDLC performance is influenced mainly by the areal surface, porous nanostructure, and pore size distribution [48]. However, EDLC materials (carbon nanotubes, activated carbon, graphene, etc.) exhibit low specific energies due to incomplete interactions between porous electrodes and electrolytes [49]. Unlike with EDLCs, pseudocapacitors operate via fast, reversible Faradaic redox reactions between an electrolyte and electrode (Figure 6b). Conducting polymers such as polyaniline (PANI) and polypyrrole (PPy) and metal oxides $\left(\mathrm{MnO}_{2}, \mathrm{NiO}_{\mathrm{x}}\right.$, etc.) are typical active materials for pseudocapacitors [50]. Pseudocapacitors deliver higher specific capacitances and larger specific energies than EDLCs. However, they offer lower cycling stabilities due to volumetric expansion and contraction during charging and discharging [51]. The third category is the hybrid supercapacitor, which uses both EDLCs and pseudocapacitor electrodes (Figure 6c) [52]. Interestingly, hybrid supercapacitors are used to deliver higher specific energies and specific power levels than either EDLC or pseudocapacitor devices [53]. As a result, many researchers have focused on the fabrication of hybrid supercapacitors using battery-type $\left(\mathrm{NiS}, \mathrm{MoS}_{2}, \mathrm{Co}_{3} \mathrm{O}_{4}, \mathrm{LiCoO}_{2}, \mathrm{LiMn}_{2} \mathrm{O}_{4}\right.$, $\mathrm{LiFePO}_{4}$, etc.) and capacitor-type electrodes [54]. These unique hybrid supercapacitors may deliver higher specific energies than EDLCs and higher specific power levels than batteries, thus satisfying demand from advancing smart devices and intelligent systems.
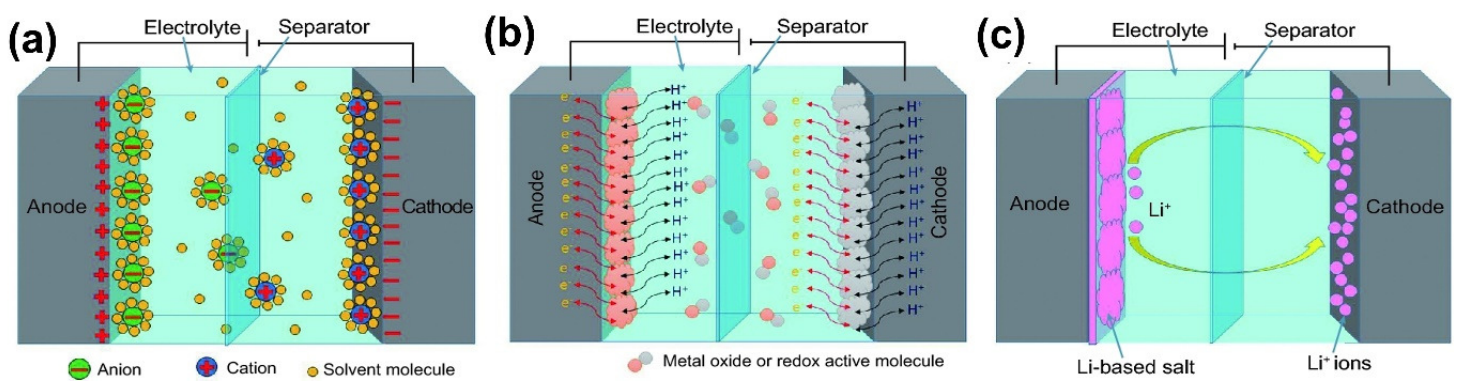

Figure 6. (a) Schematic representation of (a) EDLC, (b) pseudocapacitor (PC) and (c) hybrid supercapacitor (HSC) [54].

\subsection{Supercapacitor Electrolytes}

Choosing an electrolyte with high conductivity, high stability, and a wide stable potential is another valuable approach to improving the capacitive properties of a supercapacitor [55]. Electrolytes can be broadly categorized into liquids and solids [56]. Liquid aqueous electrolytes ( $\mathrm{KOH}, \mathrm{H}_{2} \mathrm{SO}_{4}, \mathrm{KCl}, \mathrm{LiCl}, \mathrm{NaCl}, \mathrm{K}_{2} \mathrm{SO}_{4}, \mathrm{Li}_{2} \mathrm{SO}_{4}, \mathrm{Na}_{2} \mathrm{SO}_{4}$, etc.) 
provide narrow potential windows (approximately $1.2 \mathrm{~V}$ ) and low specific energies, while non-aqueous electrolytes $\left(\mathrm{TEABF}_{4}, \mathrm{TEMABF}_{4}\right.$, acetonitrile, etc.) offer large voltage windows $(2.5 \mathrm{~V}-2.8 \mathrm{~V})$ with increased specific energies and specific power levels. However, non-aqueous electrolytes are not widely used in supercapacitors due to their many unwanted characteristics including low electronic conductivities, low specific capacitances, toxicity, and expense [57].

Recently, solid electrolytes such as poly(vinyl alcohol) (PVA), poly(methylmethacrylate), poly(acrylic acid), and poly(vinylidene fluoride-cohexafluoropropylene) have attracted increased attention among researchers due to their easy portability, insensitivity to leakage, and high flexibility. One type of solid electrolyte, the gel polymer electrolyte, contains an electrolyte in a polymer matrix and offers good electrical conductivity. In contrast, a solid polymer electrolyte contains a salt with no solvent in a polymer matrix and offers high mechanical stability but low electrical conductivity [56].

\subsection{Flexible Supercapacitors}

Progress regarding flexible supercapacitors has recently attracted considerable attention due to rapid advances in personalized electronics [58]. Due to their advantages of light weight, stretchability, portability, flexibility, and wearability, flexible supercapacitors not only decrease the volumes of electronic gadgets but also integrate them easily into human garments for flexible, wearable devices. Flexible solid-state supercapacitors (FSCs) have recently attracted substantial consideration from researchers because of their outstanding properties including small sizes, light weights, outstanding reliability, and operating temperature ranges that make them auspicious flexible energy storage systems. FSCs are generally fabricated using flexible electrodes, flexible solid-state gel electrolytes, and flexible packing materials. Thus far, substantial attention has been paid to fabricating highperformance FSCs using various electroactive materials (metal oxides, carbonaceous materials, polymers, etc.) supported on various flexible current collectors [59]. However, flexible supercapacitors face challenges such as poor mechanical robustness, low specific energies, and poor plastic current collector conductivity. This affects the ability to market these flexible systems for practical applications [60]. Hence, it is essential to develop FSCs that are highly flexible and light weight, that offer excellent mechanical durabilities and high energy-storage densities, and that can be fabricated via simple, cost-effective routes. Flexible, freestanding electrodes are the critical components of an FSC. They are made by depositing electroactive materials onto flexible current collectors.

\subsection{Progress in High-Energy-Density, Flexible Supercapacitors}

The energy and power density are the two factors that define flexible supercapacitor performance. Cycling stability and structural flexibility are also crucial. Supercapacitors are prized for their high power densities but deliver lower energy densities than batteries. Hence, many researchers have focused on enhancing supercapacitor energy densities via enormous advances in nanostructured materials and functional electrolytes [61-71]. Also, current collector flexibility and conductivity are critical to achieving high energy densities in flexible supercapacitors. Moreover, cost-effective novel device manufacturing techniques have been 
adopted to provide good architectural flexibility. Hence, proper selection of electroactive materials and current collectors can be combined with smart design of device structures and fabrication methods to substantially affect the energy densities of supercapacitors [61-68]. In 2014, Yang et al. developed a cost-effective, high-performance, solid-state flexible ASC by assembling $\alpha-\mathrm{MnO}_{2}$ nanowires (NWs) and amorphous $\mathrm{Fe}_{2} \mathrm{O}_{3}$ nanotubes (NTs) on a flexible carbon fabric with a PVA-LiCl gel electrolyte [61]. The resulting flexible $\mathrm{MnO}_{2} \mathrm{NW} / / \mathrm{Fe}_{2} \mathrm{O}_{3}$ ASC delivered a high energy density of $0.55 \mathrm{mWh} \mathrm{cm}^{-3}$ and good flexibility. Grote et al. assembled an ASC using free-standing 3D core-shell nanotube arrays of $\mathrm{SnO}_{2} / \mathrm{PPy}$ and $\mathrm{SnO}_{2} / \mathrm{MnO}_{2}$ electrode materials with $\mathrm{Na}_{2} \mathrm{SO}_{4}$ as the electrolyte and achieved a high specific energy of $27.2 \mathrm{Wh} \mathrm{kg}^{-1}$ at $0.85 \mathrm{~kW} \mathrm{~kg}^{-1}$ [62]. In 2015, Chen et al. achieved a high energy density of $99.26 \mathrm{Wh} \mathrm{kg}^{-1}$ by developing a flexible all-solid-state ASC that used carbon clothsupported $\mathrm{NiMoO}_{4} / \mathrm{PANI}$ and active carbon (AC) electrodes and a PVA-KOH gel electrolyte [63]. In 2016, Qiu et al. prepared a flexible, high-energy-density $\left(3.6 \times 10^{-2} \mathrm{mWh} \cdot \mathrm{cm}^{-2}\right)$ solid-state ASC by sandwiching Dacron cloth-supported $\mathrm{Cu}(\mathrm{OH})_{2}$ nanobelt arrays (positive electrode) between two carbon nanofiber (CNF) matrices (negative electrodes) and using KOH-PVA as the gel electrolyte [64]. In 2017, Zhu et al. achieved a high energy density of $46 \mathrm{Wh} \mathrm{kg}^{-1}$ by developing wearable, high-performance symmetric supercapacitors (SSCs) based on silver-sputtered textiles using a $\mathrm{FeCo}_{2} \mathrm{~S}_{4}-\mathrm{NiCo}_{2} \mathrm{~S}_{4}$ composite and PVA-KOH gel electrolyte [65]. In 2018, Gopi et al. utilized flexible copper- and nickel-coated polyester fabrics as current collectors for high-energy density (80.2 $\mathrm{Wh} \mathrm{kg}^{-1}$ ) SSCs that used a composite of $\mathrm{CoFe}_{2} \mathrm{Se}_{4}$ nanorods on $\mathrm{CoNiSe}_{2}$ microspheres as the electrode material and $\mathrm{KOH}$ as the aqueous electrolyte [66]. In 2019, Liu et al. constructed a flexible asymmetric supercapacitors (ASC) using carbon cloth-supported $\mathrm{Co}_{9} \mathrm{~S}_{8} @ \mathrm{NiCo}_{2} \mathrm{O}_{4}$ nanobrushes and $\mathrm{AC}$ as the positive and negative electrodes, respectively, with PVA-KOH as the gel electrolyte [67]. The resulting ASC device exhibited a high energy density (86 $\mathrm{Wh} \mathrm{kg}^{-1}$ ), outstanding cycling stability, and flexibility.

To enhance supercapacitor energy densities, substantial efforts have been made to fabricate hybrid supercapacitors (HSCs) by developing high-performance battery and supercapacitor materials [64,68]. For instance, in 2020, Zhao et al. fabricated a carbon clothsupported flexible $\mathrm{HSC}$ by combining NiCoP nanoflake-surrounded CNT nanoarrays (NiCoP/CNT) with N-doped, carbon-coated CNT nanoarrays (CNT@N-C) as battery-type and supercapacitor electrode materials, and applying a PVA-KOH gel electrolyte [68]. The as-developed flexible HSC device exhibited a high energy density of $138.7 \mathrm{Wh} \mathrm{kg}^{-1}$, which is clearly superior to those of recently developed ASC or SSC devices and indicates potential for practical application. Recently, Qiu et al. synthesized a flexible, solid-state HSC composed of phosphate ions, an oxygen-deficient modulated nickel cobaltite nanowire $\left(\mathrm{PNCO}_{x}\right.$ $\mathrm{NW}$ ) cathode, an as-prepared 3D porous graphene gel (3DPG) anode, and a PVA-LiCl gel electrolyte [64]. The as-fabricated $\mathrm{PNCO}_{x} / / 3 \mathrm{DPG}-\mathrm{HSC}$ exhibited a relatively high maximum energy density of $211 \mathrm{~W} \mathrm{~h} \mathrm{~kg}^{-1}$, which is better than recently reported SSC and ASC devices and batteries [61-67,69,70]. The present encouraging results may offer valuable insights into synthesis of high energy density HSC devices for practical applications in the near future.

These advantages and characteristics indicate that high-performance supercapacitors are the most promising, suitable candidates for effective storage of energy harvested from 
TENG devices. As a result, SCPS design and development via integration of energyharvesting TENGs and energy-storing supercapacitor technologies is highly desirable and is reviewed in the sections below.

\section{Bio-Inspired SCPS (Integration of TENGs with Supercapacitors)}

The output from a TENG cannot be utilized to operate most electronic gadgets directly, as they require consistent DC power sources. Hence, integration of an energy storage unit is crucial to real-time TENG usage [71]. Supercapacitors have attracted considerable attention compared to other energy storage technologies and are well matched with TENGs because of their high specific power capabilities, rapid charge-discharge characteristics, long cycle lives, and environmental adaptabilities. However, these two devices always function separately and rely on distinct phenomena that inhibit their use as viable power sources for electronic devices. Therefore, SCPS development requires direct integration of energyharvesting TENGs with energy-storing supercapacitors [35]. Here, we discuss recent progress in SCPSs that integrate TENGs with supercapacitors in various configurations.

\subsection{Flexible, Paper-based SCPSs}

For light-weight, flexible, portable SCPSs with high charge outputs, Yang et al. fabricated a cost-effective, lightweight, and environmental friendly, paper-based TENG based on origami configurations for harvesting ambient mechanical energy and serving as a selfpowered pressure sensor. As fabricated paper-based TENG can act as a self-powered pressure sensor to sense the difference in mass of standard coins [72]. In another work, Yang et al. developed a new type of flexible TENG by assembling serpentine-patterned electrodes and a wavy-structured Kapton film [73]. As developed flexible TENG could be conveniently attached onto any part of a human body for self-powered body motion sensing. Recently, Sun et al. developed an ultralight, flexible SCPS using an energy-harvesting all-electrospun paper-based TENG and energy-storing all-electrospun paper-based supercapacitor [74]. The corresponding schematic is shown in Figure 7a. The design relies on development of electrospun, paper-based TENGs and supercapacitors that contain one insulating and one conducting flexible electrospun paper sheet, respectively. In this flexible SCPS design, mechanical energy from human body movement is converted into electrical energy using an electrospun paper-based TENG. Supercapacitors can store the resulting AC energy and sustainably operate wearable or portable electronics. When the devices were fabricated separately using electrospun paper, as-fabricated single electrospun paper-based TENG delivered a $\mathrm{V}_{\mathrm{OC}}, \mathrm{I}_{\mathrm{SC}}, \mathrm{Q}_{\mathrm{SC}}$, and maximum average power density of $\sim 98.6 \mathrm{~V}, 11.3 \mu \mathrm{A}, \sim 31.1$ $\mathrm{nC}$, and $18.4 \mathrm{~mW} / \mathrm{m}^{2}$, respectively, at an $8 \mathrm{~Hz}$ frequency of motion. A single electrospun paper-based supercapacitor unit exhibited an outstanding specific capacitance $\left(150 \mathrm{~F} \mathrm{~g}^{-1}\right)$ and good cycle life. To explore potential SCPS applications, three electrospun paper-based TENGs and three supercapacitors were attached in parallel and series to form SCPSs, as shown in Figure 7b. Before charging the electrospun paper-based supercapacitor, the electrospun paper-based TENG AC current was converted into DC current using a bridge rectifier (inset of Figure 7c). Figure 7c depicts the charging plot of the SCPSs at $5 \mathrm{~Hz}$ frequency for real-time applications. The electrospun paper-based supercapacitor can be 
charged proportionally in accordance with the maximum $\mathrm{V}_{\mathrm{OC}}$ using the rectified output from the electrospun, paper-based TENG. The system needs approximately $2150 \mathrm{~s}$ (approximately $36 \mathrm{~min}$ ) to charge the electrospun paper-based to $2 \mathrm{~V}$. The as-developed SCPSs can drive a calculator and an electronic watch.

Recently, Guo et al. developed an ultralight cut-paper-based SCPS that could efficiently harvest and store energy by integrating a cut-paper-based TENG with a paperbased supercapacitor [75]. A schematic of the all-paper-based SCPS is shown in Figure 7d. An ultralight, cut-paper-based SCPS is established by integrating a cut-paper-based TENG and paper-based supercapacitor using a rectifier. As-developed SCPS fits in a wallet for practical applications (Figure 7e). The V-t profile was recorded as a hand moved at various frequencies. The result is shown in Figure $7 \mathrm{f}$. As shown in the inset of Figure $7 \mathrm{f}$, two times increase of short-circuit charge transfer $\left(\mathrm{Q}_{\mathrm{sC}}=1350 \mathrm{nC}\right)$ can be produced and used to charge the serially connected paper-based supercapacitors to $1.38 \mathrm{~V}$ during each contact-separation cycle. This indicates that the PC-SCPS charging strategy is effective. For real-time applications, a temperature sensor and electric watch are operated using as-fabricated, cutpaper-based SCPSs as a sustainable power source.

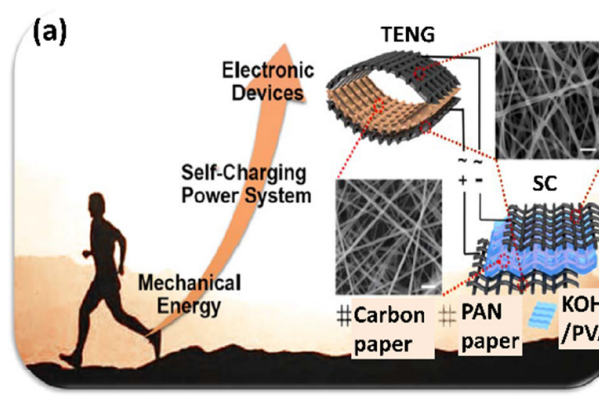

(b)
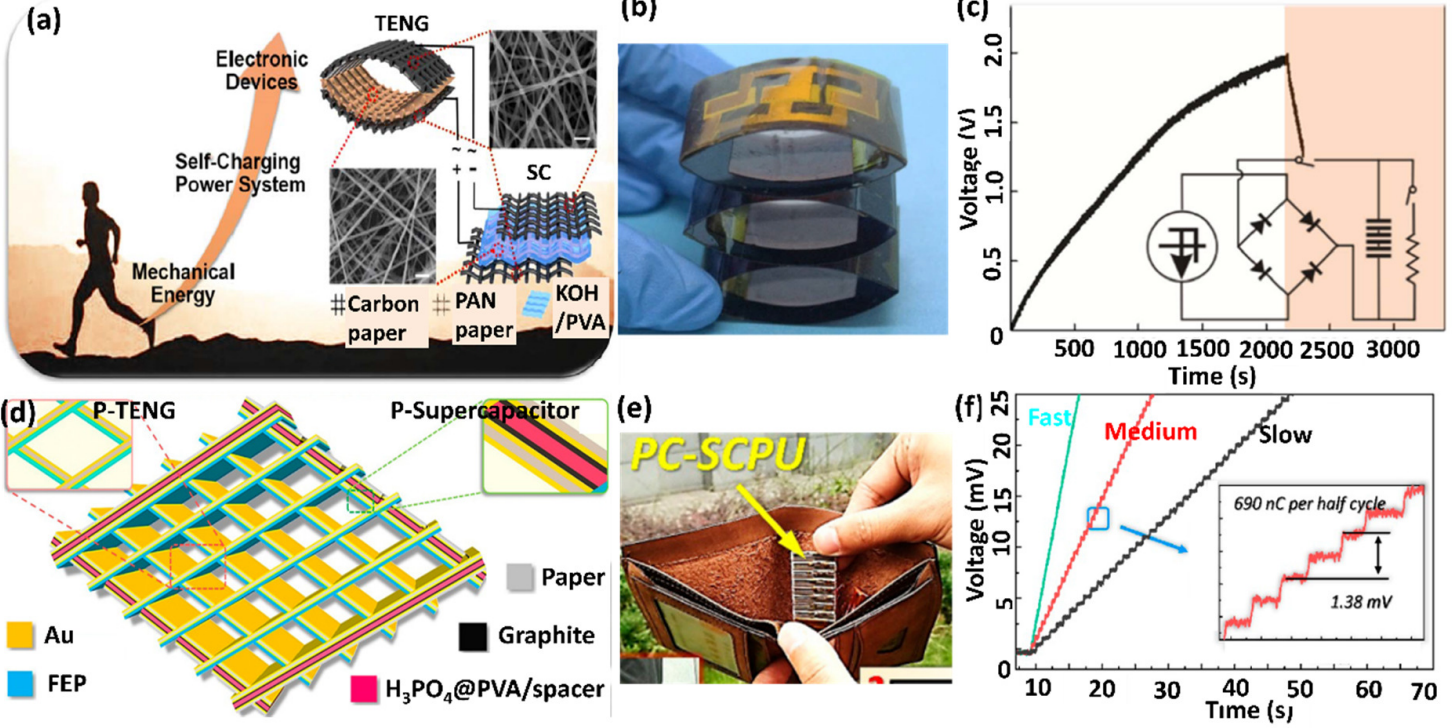

Figure 7. (a) Schematic demonstration of the all flexible electrospun papers based SCPS. (b) Photograph and (c) voltage profile of an as-developed SCPS composed of 3 electrospun paper based TENG in parallel and 3 electrospun paper based supercapacitors in series. Source: Sun et al. [74]. Reprinted with permission of Elsevier. (d) Schematic demonstration of the all-paper-based cut-paper SCPS. (e) Photograph of a cut-paper SCPS attached to a wallet. (f) Voltage-time plot of the cut-paper SCPS (charged by hand flapping from slow process to rapid process). Source: Guo et al. [75]. Reprinted with permission of ACS publications. 


\subsection{Flexible Fiber- and Textile-based SCPSs}

Flexible SCPSs have recently played important roles in energy harvesting and selfpowered, wearable electronics. In 2015, Wang et al. first introduced a flexible, wearable SPCS comprised of integrated fibre supercapacitors and a fiber-based TENG [76]. The fibrebased SCPS was developed by integrating three fibre supercapacitors with a fibre-based TENG using a rectifier. The FSC and FTENG were integrated via a rectifier and the three series-connected fibre supercapacitors could be charged to $2.5 \mathrm{~V}$ in $873 \mathrm{~s}$ using the rectified output of a fibre-based TENG and discharged at $1.4 \mu \mathrm{A}$ over $258 \mathrm{~s}$.

Later, $\mathrm{Pu}$ et al. performed simple, extensible development of a supercapacitor made from solid-state, flexible yarn and integrated it with a TENG cloth to form an SCPS [77]. The SCPS contained the energy-harvesting TENG fabric, energy-storing supercapacitor fabric, and wearable electronics. The rGO-Ni yarns were prepared by depositing a rGO film and $\mathrm{Ni}$ layer onto a polyester yarn surface. A symmetric yarn-type supercapacitor was fabricated by assembling two rGO-Ni yarns with $\mathrm{PVA}-\mathrm{H}_{3} \mathrm{PO}_{4}$ gel as the solid electrolyte and separator. In the TENG cloth, Ni-coated strips constituted one electrode, while strips of Ni-coated parylene were the other. A single symmetric yarn supercapacitor delivered a high specific capacitance (13 $\mathrm{mF} \mathrm{cm}^{-1}$ ) and excellent cycle life (96\% after 10,000 cycles). The TENG could charge the three series-connected yarn supercapacitors to $2.1 \mathrm{~V}$ in $2009 \mathrm{~s}$ at $5 \mathrm{~Hz}$. The device could discharge at $1 \mu \mathrm{A}$ for $811 \mathrm{~s}$, further revealing the validity of the as-fabricated SCPSs. Zhao et al. developed a novel SCPS composed of flexible asymmetric supercapacitors and a flexible TENG [78]. Supercapacitors were fabricated using metal-organic framework (MOF)-derived nanowire hybrid carbon nanotube fibers. In addition, $\mathrm{Cu}$-coated and flexible polytetrafluoroethene (PTFE) film-Cu coated electrodes were combined into a TENG using Kapton film. The flexible, fibre-shaped MOF-derivative hybrid array asymmetric supercapacitor was combined with a TENG via a rectifier to form an SCPS designed for concurrent energy harvesting and storage. Xiong et al. fabricated a flexible SCPS by integrating a flexible asymmetric supercapacitor with a TENG [79]. A high-efficiency, flexible, solid-state asymmetric supercapacitor was fabricated from core-shell peptide-Cog $\mathrm{S}_{8}$ nanobricks and activated carbon. The resulting asymmetric supercapacitor could be integrated with a TENG to form a flexible SCPS. The TENG charged the supercapacitor continuously for $2.7 \mathrm{~h}$ so that it could drive a red LED for $21 \mathrm{~min}$, thus illustrating its great impact on future wearable electronics applications.

E-textiles use electronics with multifunctional attributes to make trendy clothing that offers new insights into further advances in wearable electronics. Combining fabric-based TENGs and supercapacitors into a single textile material is important to E-textile progression. These SCPSs can be woven directly into clothing and can be used to convert human movement into electrochemical energy. Based on conventional weaving strategies, Chen et al. developed a SCPS composed of a free-standing fabric TENG and a woven supercapacitor [80]. This SCPS harvested energy from daily human activities (running, walking, and cycling) and stored the resulting energy using the woven supercapacitor. Free-standing mode fabric TENGs were prepared on textiles that contained woven cotton, PTFE wires, and carbon. Each symmetric woven supercapacitor used the carbon fibre as the electrode and the 
$\mathrm{RuO}_{2}$ on the carbon fibre as the electroactive material. The cotton threads were the separators and $\mathrm{PVA}-\mathrm{H}_{3} \mathrm{PO}_{4}$ was the electrolyte. Using human movements, the rectified output from the free-standing mode fabric TENG was stored in the woven supercapacitor and then utilized in practical applications such as operating an electric watch.

\subsection{Yarn Based SCPSs}

Textile production technologies can provide a variety of textile-based SCPS manufacturing paths that allow the products to adapt to various intricate mechanical deformations. Highly stretchable, wearable, flexible, fully shape-adaptive textile-based SCPSs are highly desirable due to the exceptionally tough operating circumstances (stretching, bending, and twisting) of wearable devices. As a result, various types of woven self-charging power fabrics have recently been described for wearable electronics [76,77]. However, interlacing yarns operate straight horizontally and vertically that results in difficult to maintain the high stretchable efficiency of woven fabric, which its application real time. In contrast, knitted fabric structures exhibit excellent elasticities due to the presence of various interdependent, successive rows of interrelated yarn loops. Also, knitted fabrics can be stretched in all directions because of the space present in each loop.

Dong et al. fabricated a highly stretchable all-yarn-based SCPS that is quite effective at energy harvesting and storage of energy from human body movements (Figure 8a) [81]. The SCPS is constructed by combining an all-yarn based TENG energy harvesting unit with an energy-storing, all-solid-state yarn supercapacitor. The TENG is developed by coating a three-ply twisted stainless steel-polyester fibre-coupled yarn surface with silicone rubber. The all-solid-state symmetric supercapacitor is assembled using two parallel PEDOT:PSS/carbon nanofiber/carbon fibre (CF) electrodes and an $\mathrm{H}_{3} \mathrm{PO}_{4}$-PVA gel electrolyte. The CNF and PEDOT:PSS are coated onto the CF via dip-coating. The wearable SCPS is fabricated by knitting the as-prepared yarn supercapacitor into the TENG fabric. Upon hand tapping, the harvested rectified output of the TENG fabric is stored in the two-series yarn supercapacitor (Figure $8 \mathrm{~b}$ ). When $\mathrm{S} 1$ is on and $\mathrm{S} 2$ is off, the yarn supercapacitor voltage increases linearly and the device can drive a calculator continuously for $260 \mathrm{~s}$ (blue plot in Figure 8c). If both S1 and S2 are on, the voltage reaches a threshold and the device can operate a temperaturehumidity meter (red plot in Figure 8c). 


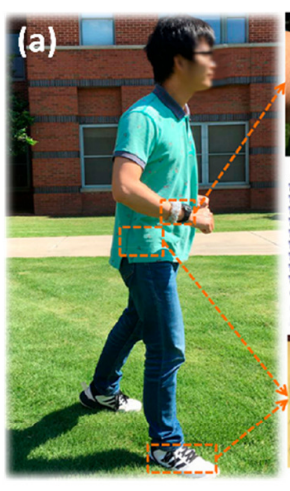

(d)
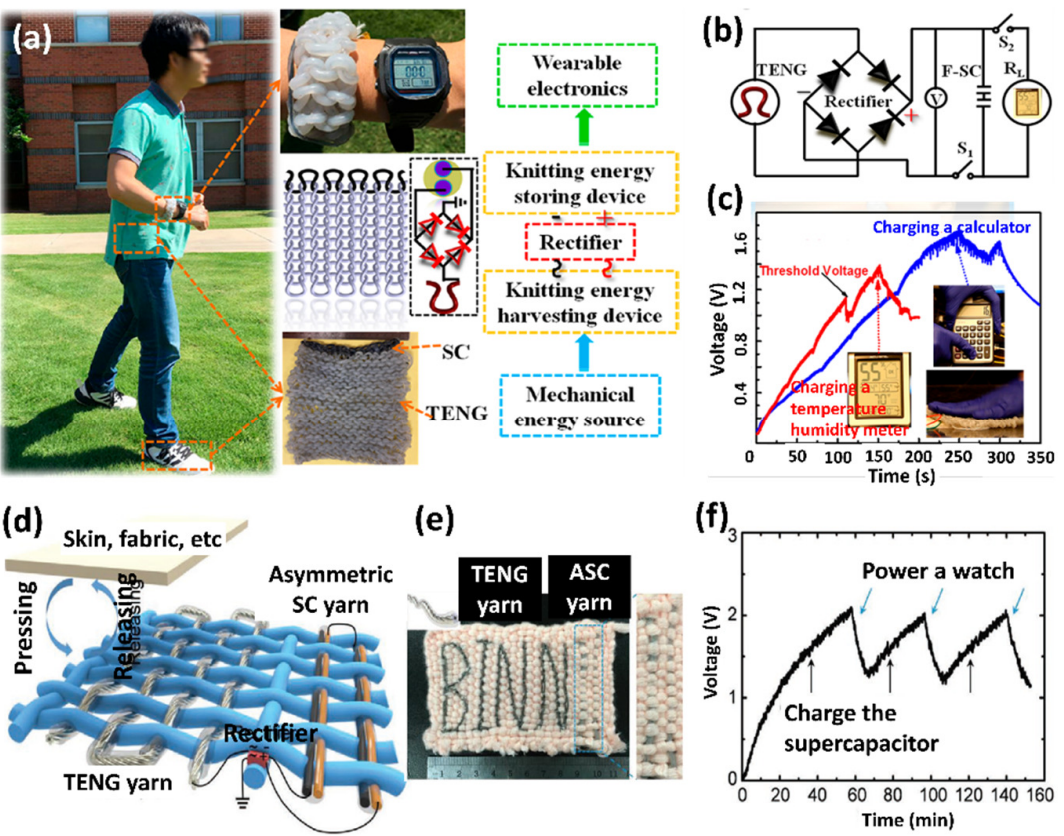

Figure 8. (a) Scheme and (b) equivalent circuit of an all-yarn-based SCPS consists of fiber TENG and supercapacitor. (c) Charging plots of two-series supercapacitors by manually tapping the TENG fabric. Source: Dong et al. [81]. Reprinted with permission of ACS publications. (d) Schematic illustration, (e) photograph, and (f) voltage profile of the asfabricated self-charging power textile. Source: Liu et al. [82]. Reprinted with permission of John Wiley \& Sons.

Liu et al. recently fabricated a SCPS by combining an energy-harvesting yarn-based TENG with an energy-preserving, yarn-based asymmetric supercapacitor [82]. Initially, conductive yarns were prepared via successive coating of a common polyester yarn surface with $\mathrm{Ni}$ and $\mathrm{Cu}$ films. The yarn-based asymmetric supercapacitor is fabricated by assembling yarn electrodes. The hydrothermally self-assembled rGO/CNT coating is used to make the anode, while an electroplated $\mathrm{Ni}-\mathrm{Co}$ bimetallic oxyhydroxide ( $\mathrm{NiCo} \mathrm{BOH}$ ) coating forms the cathode. The yarn-based TENG is prepared using PDMS-coated conductive yarns. Figure 8d provides a schematic illustration of the SCPS, in which the yarn-based TENG and asymmetric supercapacitor are bridged via a rectifier. As shown in Figure 8e, the SCPS is composed of "BINN"-shaped TENG yarns and two series-connected asymmetric supercapacitor yarns. The resulting SCPS can operate wearable electronics. The energy harvested from a yarn-based TENG can charge the yarn asymmetric supercapacitors at $4 \mathrm{~Hz}$ to operate an electronic watch (Figure 8f). Over $58 \mathrm{~min}$, the yarn asymmetric supercapacitor voltage reaches $2.0 \mathrm{~V}$, which allows it to drive the watch for $5 \mathrm{~min}$. Although continuous power supply is not achieved, this approach may be applicable to portable electronics that only function occasionally.

\subsection{Sandwich-Structured SCPSs}

Physical vapour-deposited $\mathrm{Cu}$ and $\mathrm{Al}$ metals are typical TENG electrode materials but oxidize and corrode easily in difficult weather [83]. In contrast, electrically conducting 
polymers (ECPs) are promising materials and triboelectric layers that can be fabricated quickly via facile, cost-effective electrochemical deposition routes. The ECP polypyrrole (PPy) is a potential supercapacitor electrode material because of its good electrical conductivity, decent chemical and thermal stabilities, simple preparation, cost-effectiveness, and biocompatibility [84].

Most SCPS devices contain separate energy harvesting and storage units that reduce their overall specific energies and limit device applications. Therefore, it is highly desirable to fabricate single-unit energy harvesting and storage systems. In 2016, Wang et al. developed an all-plastic, sandwich-structured SCPS using PPy polymer-based TENGs and supercapacitors [35]. Here, the TENG contained a triboelectric layer with conventional cauliflower-like PPy (cPPy) as the back electrode and hollow PPy with a hornlike morphology (hPPy) as the triboelectric electrode. Supercapacitors were fabricated via assembly of two hPPy electrode materials using a porous polyethylene (PE) film separator and $\mathrm{H}_{3} \mathrm{PO}_{4}$-PVA gel electrolyte. To improve output, several TENGs (typically three) and supercapacitors (typically four) were attached in parallel and series. The TENGs and supercapacitors were stacked together to form a sandwich structure. The supercapacitors were charged from $10 \mathrm{mV}$ to $40 \mathrm{mV}$ over $30 \mathrm{~s}$ by tapping the as-prepared SCPSs with human fingers and could drive an electronic device sustainably.

Song et al. fabricated an integrated, sandwich-structured SCPS composed of PDMSbased two-arch-shaped TENGs with a packaged CNT/paper-based solid-state supercapacitor [85]. Two TENGs were attached in parallel to improve the charge storage performance. Upon application of $8 \mathrm{~Hz}$ ambient vibrations to the SCPS, the rectified TENG output charged a supercapacitor to $900 \mathrm{mV}$ in $3 \mathrm{~h}$. Once they were completely charged, three built-in, serially connected SCPSs consistently operated a calculator. Recently, Deka et al. fabricated a woven carbon fibre-based multifunctional sandwich-shaped SCPS device, which included a single TENG and a supercapacitor [86]. The woven carbon fibre surface was coated with P-doped, branched $\mathrm{Cu}-\mathrm{Mn}$ selenide nanowires via a two-step hydrothermal method. A facile, low-cost vacuum assisted resin transfer molding (VARTM) route was used to develop a P@Cu-Mn selenide-woven carbon fibre-based supercapacitor. A positive TENG electrode was prepared by spin coating PDMS onto P@Cu-Mn selenide-woven carbon fiber. Finally, a sandwichshaped SCPS was developed by integrating the supercapacitor and TENG electrodes. When the two dielectric layers came into contact, the rectified TENG output charged the supercapacitor. The integrated TENG produced $7.4 \mathrm{~W} \mathrm{~m}^{-2}$ of power and the supercapacitor exhibited a high specific energy $\left(97.21 \mathrm{Wh} \mathrm{kg}^{-1}\right)$ and high specific power $\left(54.25 \mathrm{~W} \mathrm{~kg}^{-1}\right)$. After patting the device for $70 \mathrm{~s}$, the supercapacitor was charged to $19.2 \mathrm{~V}$ by the rectified output of the highly efficient TENG and could operate 60 serially connected LED bulbs.

\subsection{Package-Structured SCPSs}

In the above section, TENGs and supercapacitors were integrated into single SCPS devices. However, these structures suffer from drawbacks. For example, the separate parts of the TENG and supercapacitors are connected a using rectifying circuit, which requires external wiring that adds volume to the system. On the other hand, proper sealing of conventional supercapacitors is essential due to the potential for liquid electrolyte 
evaporation. Moreover, TENGs require spacers due to their contact-separation operating modes. This makes developing integrated, efficient units complex. To address these issues, Yi et al. developed a washable, waterproof, stretchable SCPS that effortlessly integrated a stretchable TENG, stretchable supercapacitor, and rectifier into a single monolithic package with silicone rubber [87]. Carbon black was coated onto silicone rubber as a stretchable TENG electrode material, while the supercapacitor comprised two stretchable polypyrrolecarbon black electrodes with a $\mathrm{PVA}^{-} \mathrm{H}_{3} \mathrm{PO}_{4}$ gel electrolyte. The as-fabricated stretchable SCPS extracted energy from various human movements using various mechanical deformations (e.g., bending, stretching, and twisting) via the TENG, stored the energy in a supercapacitor, and then drove an electronic watch. Guo et al. fabricated a package-shaped whole-shape-adaptive SCPS for wearable electronics [88]. Based on the concept of kirigami architecture, an ultra-stretchable kirigami paper-based supercapacitor was prepared with $100 \%$ stretchability and a $1 \mathrm{mF} \mathrm{cm^{-2 }}$ specific capacitance. An ultra-stretchable, shapeadaptive TENG was prepared using Ag nanowires and silicon rubber that delivered an output of $160 \mathrm{nC}$ per half operating cycle. An all-in-one shape-adaptive SCPS was developed by integrating the supercapacitor into the TENG via a full-wave rectifier. The resulting SCPS harvested energy from hand movement and continuously operated an electronic watch.
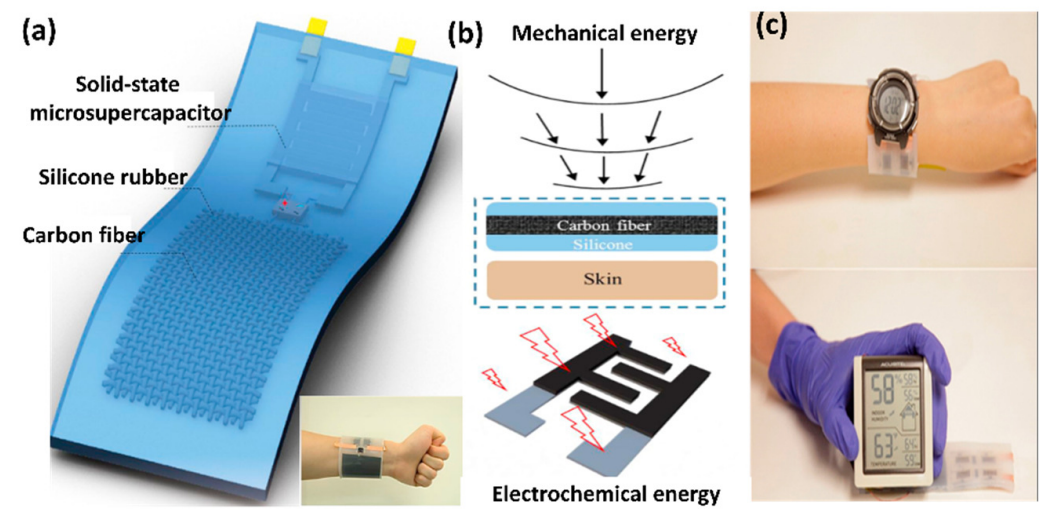

Figure 9. (a) Schematic demonstration and (b) mechanism of SCPS package with a TENG and a MXene-based MSC. (c) Practical application of SCPS package. Source: Jiang et al. [92]. Reprinted with permission of Elsevier.

Research on all-solid-state microsupercapacitors has recently attracted considerable attention because of rapid development of miniaturized electronic gadgets and IOT devices [89]. Microsupercapacitors offer high rate capabilities, high specific power levels, long cycle lives, and environmental friendliness. Due to their high rate capabilities and rapid responses, energy-storing microsupercapacitors can be effectively integrated with energy-harvesting TENG units. Luo et al. used the easy, low-cost laser engraving approach to develop a flexible SCPS composed of a TENG integrated with a microsupercapacitor array and rectifier [90]. Upon applying ambient stress to the as-developed SCPS, the microsupercapacitors were charged to $3 \mathrm{~V}$ in 117 min using the rectified TENG output. Wang et al. developed a bioinspired, stretchable TENG using physiological saline as the electrode and a silicone rubber encapsulation and triboelectric layer [91]. Upon integrating the TENG and the 
microsupercapacitor, the two units distributed a single solution that acted as both the TENG electrode and the microsupercapacitor electrolyte. A stretchable SPCS was prepared by combining a TENG, a microsupercapacitor, and a rectifier using a single sodium sulphate electrolyte. Since the rectifier and microsupercapacitor areas were small compared to the full SCPS area and these two units were situated on the device edge, the resulting SCPS exhibited high stretchability and conformability. Upon patting the device, the potential of the SCPS with four microsupercapacitors in series reached $1.16 \mathrm{~V}$ within $6.5 \mathrm{~s}$. Jiang et al. fabricated a highly compact single monolithic package SCPS by integrating a TENG with MXene-based microsupercapacitors [92]. Figure 9a provides a schematic illustration of a SCPS composed of a TENG, rectifier, and MSC. These are encapsulated using stretchable dielectric silicone rubber, resulting in a prolonged MSC lifetime with no electrolyte leakage or evaporation. Upon contact with human skin, the MSCs start to store charge using rectified TENG output (Figure 9b). The as-developed SCPSs operate a digital watch and a thermo-humidity meter $(1.5 \mathrm{~V})$ for a few minutes (Figure $9 \mathrm{c}$ ).

\subsection{Hybrid SCPSs}

Most SCPS are developed by integrating a TENG energy harvesting unit with an energy-storing supercapacitor unit. However, rather little energy is harvested from the TENG alone. To improve the energy-harvesting efficiency, the TENG should be connected to other energy harvesting technologies to produce a hybrid energy-harvesting device. Hybrid SCPSs with hybrid energy harvesting devices may be an efficient strategy for providing energy to large electronics and therefore expanding the practical application of these systems. Thus, Wen et al. fabricated a fabric-hybridized SCPS that not only harvested solar energy from sunlight dye-sensitized solar cells, but also generated mechanical energy from random human body motion (TENG) and then transferred that energy to a supercapacitor [93]. The fabrichybridized SCPSs composed of three functional devices, including fibre-shaped dyesensitized solar cells and fibre-shaped TENGs for solar and mechanical energy, respectively, plus fibre-shaped supercapacitors for energy storage. The solar cells and supercapacitors are connected to form a single fibre-shaped TENG unit that scavenges energy from body motion. The fabric-hybridized SCPS is connected to a T-shirt to harvest solar and motion-based energies from daily outdoor and indoor activities. From the equivalent circuit of the fabrichybridized SCPS, the rectifier converts the TENG AC output to DC output to charge the fibre-shaped supercapacitors and a diode blocks current flow from the TENG to the solar cells. Charging the fibre-shaped supercapacitors to $1.8 \mathrm{~V}$ using the solar cells alone limits their reliability and practicability (light blue-shaded area), but the TENG charges the supercapacitors continuously to the maximum voltage via the solar cells.

Both piezoelectrics and TENGs have been considered as a substantial energy sources for hybrid SCPSs. Proper integration of piezoelectrics and TENGs helps to enhance energy harvesting capacities via their similar output behaviours, matching impedances, and easy preparation methods [94]. Qin et al. developed a flexible, compact, smart hybrid SCPS package by combining a hybrid piezoelectric/TENG and a solid-state electrochromic MSC array [95]. The electrochromic MSC utilized interdigitated ITO electrodes with Ag nanowires and $\mathrm{NiO}$ to deliver a high specific capacitance $\left(3.47 \mathrm{mF} \mathrm{cm} \mathrm{cm}^{-2}\right.$ ) and excellent cycling 
behaviour ( $80.7 \%$ over 10,000 cycles). In the hybrid piezoelectric/TENG, the piezoelectric nanogenerator and TENG shared the same silver electrode and achieved a large output voltage and enhanced current of $150 \mathrm{~V}$ and $20 \mu \mathrm{A}$, respectively. The hybrid SCPS package integrated an arch-shaped hybrid piezoelectric/TENG with a nine-microsupercapacitor array (with a combination of parallel and series connections). Upon continuous impact of a palm on the hybrid piezoelectric/TENG, the microsupercapacitors were charged from 0.05 to $1 \mathrm{~V}$ in $260 \mathrm{~s}$. The voltage across the three serially connected electrochromic microsupercapacitors reached a maximum of $3 \mathrm{~V}$ in $300 \mathrm{~s}$ using the hybrid piezoelectric/TENG under continuous palm impact. To demonstrate a practical application, the nine MSCs operated an LED.

\section{Power Management}

Typically, TENGs provide large output voltages and low output currents that do not meet electronics requirements. Also, TENGs have high inherent impedances that result in low-efficiency energy conversion. The purpose of an SCPS is to store TENG-harvested energy in an energy storage unit. Conventionally, a bridge rectifier is used to convert the harvested TENG AC energy into DC energy for storage in an energy storage unit that powers electronics. However, direct integration of TENG and supercapacitor units via a bridge rectifier provides low charging efficiency and high energy loss because of the huge impedance difference between the two components. The TENG devices have large internal impedances, but supercapacitors have small impedances [96]. Therefore, it is important to design and develop power management circuits that address this issue in order to achieve high charging efficiencies.

In 2014, to improve the charging efficiency of a TENG-based power-supply, Zhu et al. designed a power management circuit composed of a transformer, a rectifier, a voltage regulator and capacitors [97]. The transformer could dramatically increase the output current per unit of output voltage, thus significantly decreasing the output impedance of the TENG and resulting in high charging efficiency. Bhatia et al. developed a mechanical frequency regulator that could produce predictable electric output from the TENG despite changes in the external input [98]. In the power management circuit, the mechanical frequency regulatorTENG was directly attached to the power transformer and the rectified output from the transformer charged a capacitor. Upon regulating the TENG output at $50 \mathrm{~Hz}$, the energy stored in the capacitor with the transformer was 2.5 times higher than that without the transformer. The transformer was not compatible with the lateral-sliding-mode TENG due to the low mechanical energy frequency and large power losses that occur when the operating frequency varies [99]. In contact-separation mode, it is much more difficult to manage a randomly pulsed waveform [100]. Accordingly, there is an immediate need for a universal power management strategy that is applicable to both contact-separation and lateral-sliding TENG modes. Thus, Niu et al. proposed a two-stage power management circuit for SCPSs that can generally be employed with all types of TENGs with pulsed outputs [101]. The asdeveloped self-charging system was composed of a TENG, a power management circuit, and a low-leakage energy storage device. However, $25 \%$ of the energy loss was generated when energy was transferred from the TENG to the bridge capacitor. Furthermore, this capacitor must be tuned to various TENG parameters, which is inefficient and nearly impossible. To 
address this challenge, Cheng et al. developed a power management circuit that could effectively extract the maximum harvested energy from the TENG and then transfer it to a storage unit via optimized inductor-capacitor oscillation [102]. With this power management circuit, the SCPS stored more than 2600 times the energy of a standard circuit and achieved an AC-to-DC power conversion efficiency that exceeded $72 \%$. This circuit was employed as a power source for continuous operation of various commercial electronics like LED bulbs, calculators, and pedometers.

In 2017, Xi et al. proposed a universal TENG power management strategy that could enhance the energy conversion efficiency using DC buck power conversion and self-control techniques [103]. The power management module comprised a TENG, a rectifier, a traditional DC-DC buck converter, and an energy storage unit. At a minimum frequency of 1 $\mathrm{Hz}$, the matching TENG impedance changed from $35 \mathrm{M} \Omega$ to $1 \mathrm{M} \Omega$ at $80 \%$ efficiency and the energy stored in the capacitor increased by 128 times. Further, Liang et al. developed a power management technique for a network of TENGs based on a rectified buck conversion circuit by combining the TENG network, rectifiers, and a classical DC-DC buck converter [104]. The TENG units in the interconnected network were connected in series to an AC-DC rectifier bridge to produce higher potentials and impedance matching with the power management module (PMM). When the PMM was implemented, the TENG network generated a constant, consistent DC voltage at the load resistor and the energy stored in the capacitor was enhanced by up to 96 times. This system could be utilized to operate a wireless transmitter and a digital thermometer.

In contrast to previous circuits that were based on inductor-based management, Tang et al. proposed an inductor-free TENG power management circuit [105]. The powertransformed-and-managed TENG was analysed by incorporating self-connected-switching capacitors in the TENG. These capacitors were arranged in series in charging mode and then connected in parallel for power output. The proposed power-transformed-and-managed TENG lowered the output voltage and enhanced the output charge with minimal energy loss. As a result, the charging time for a power-transformed-and-managed TENG-based capacitor was 8 times better than for a conventional TENG. In addition, $\mathrm{Zi}$ et al. proposed an inductorfree management design that used capacitors connected in series and parallel [106]. Moreover, $\mathrm{Zi}$ et al. designed a TENG with a motion-triggered-switch-based circuit to produce the designed charging cycle [107]. Using the V-Q plot of the TENG, they fabricated a charging cycle that used a motion-triggered switch to modulate the SCPS charge flow. Compared to a direct cycle, the as-produced charging cycle achieved a higher charging rate, increased the energy-storage efficiency by up to $50 \%$, and increased the saturation voltage by 2 times. Although this switch could maintain and improve the TENG output performance, its design made the system complex and expensive. Hence, there is a need for design and development of low-cost, highly efficient power management module strategies to further improve energy-transfer efficiency. 


\section{Summary and Perspective}

In summary, by developing analogies with self-power generation in various organisms (such as electric rays, geckos, elephant nose fish, Guiana dolphins, and electric eels.), we have reviewed the latest advancements in SCPSs and integration of energy harvesting and energy storage units. In particular, SCPSs were developed by integrating TENGs and supercapacitors. Upon collecting environmental mechanical energy, the energy harvested from the TENG charges energy-storing supercapacitors to provide a power source for multifunctional electronic devices. By integrating TENG and supercapacitor units either at the device scale via separate units or at the electrode scale along with shared electrodes, various types of SCPSs have been developed including flexible paper-based SCPSs, flexible fibre- and textile-based SCPSs, yarn-based SCPSs, sandwich-structured SCPSs, and packagestructured SCPSs. Thus, integration is an auspicious strategy for producing sustainable portable and wearable electronics that operate from foraged energy. Moreover, to enhance the TENG harvesting efficiency, hybrid SCPSs were developed that integrate the TENG with other energy-harvesting technologies such as solar cells and piezoelectric nanogenerators. Furthermore, to enhance the energy-transfer efficiency, various designs integrate power management circuits with TENGs and energy storage. Based on their superior self-charging and energy-supplying performances, continued development of high-performance SCPS devices will have a profound influence and can be applied to future wearable and portable electronics applications.

Although considerable improvement has occurred thus far, future studies are still essential to development of high-performance SCPSs that integrate energy harvesting and storage technologies. Future research should address the following situations:

(1) In SCPSs, relatively little energy is harvested from the TENG alone. This can be enhanced by connecting the TENG to other energy-harvesting technologies such as solar cells, wind energy sources, and piezoelectric nanogenerators, resulting in hybrid energyharvesting devices. To simultaneously harvest solar, wind and wave energies, we propose a hybrid SCPS that uses a hybrid energy-harvesting panel that floats on the surface of the ocean, as shown in Figure 10. To maximize the power supply, solar panels and wind turbines can be implanted alongside the TENG networks. The power generated using solar energy, wind-driven generators, and TENG networks can be transferred effectively to power plants, domestic appliances, or the electrical grid. Therefore, further development of hybrid SCPSs with hybrid-energy-harvesting devices will be an efficient strategy for meeting the needs of large electronics and expanding real-time SCPS applications. 


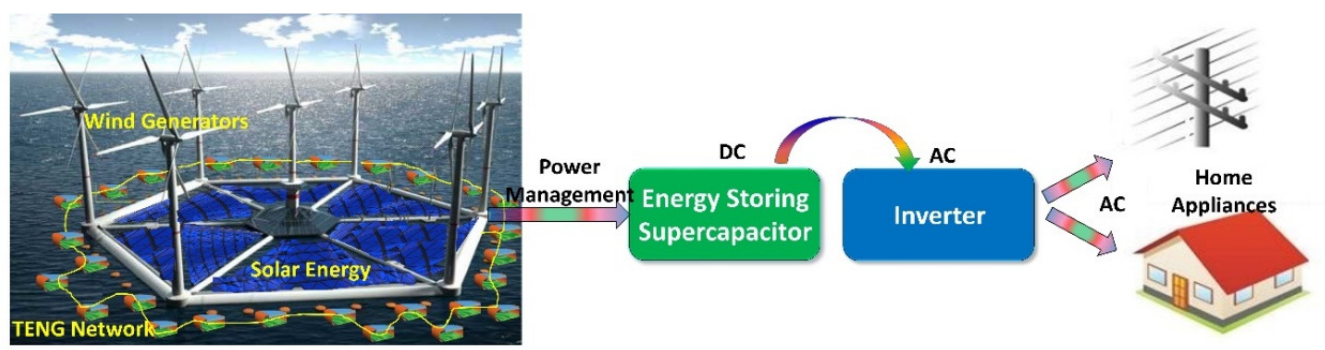

Figure 10. Hybrid SCPS installed above water surface with various integrated energy harvesting devices including solar energy, TENG network and wind generators.

(2) The performance of the supercapacitor is mainly relied on the electroactive material, structural design and fabrication methods. Hence, development of unique structures of new electroactive materials with facile fabrication strategies are highly desirable to achieve the high specific power, high specific energy, rapid charge-discharge and long cycling life. Besides, the importance of choosing the appropriate current collectors, electrolytes, separators, etc. should not be underestimated.

(3) Supercapacitors suffer from self-discharge and leakage currents, which can substantially decrease SCPS performance. Future research should focus on suppressing energy storage unit self-discharge and leakage issues.

(4) Improving and optimizing the system transfer efficiency is quite challenging. SCPSs deliver low energy conversion efficiencies because of the impedance imbalance between the energy-harvesting TENG and energy-storing supercapacitor. Hence, the impedances and specific capacities of supercapacitor units must balance the pulsed TENG output for higher energy-transfer efficiency.

(5) SCPS safety, cost, environmental friendliness, and ease of integration are important concerns. Future SCPS design and development should address miniaturization, diversification, integration, and portability. Moreover, developing a SCPSs with excellent flexibility, highly stretchable and waterproof are also a significant and most promising research pathway for next-generation portable and wearable smart electronics.

(6) Mostly reported SCPS design composed of separate units of harvesting, storage and rectifier devices, which not only reduce the specific power, specific energy and stability but also with and external wiring for electrical connection results extra volume to the system and limit their applications. Hence, the research on the SCPS design composed of single unit of harvesting, storage and rectifier devices is highly desirable for practical applications.

\section{Acknowledgements}

King Abdullah University of Science and Technology (KAUST) baseline funding, BAS/1/1614-01-01. 


\section{References}

[1] Z.L. Wang, Adv. Mater., 24, 280-285 (2012). https://doi.org/10.1002/adma.201102958

[2] J.B. Goodenough, K. Park, J. Am. Chem. Soc., 135, 1167-1176 (2013). https://doi.org/10.1021/ja3091438

[3] G. Wang, L. Zhang, J. Zhang, Chem. Soc. Rev., 41, 797-828 (2012). https://doi.org/10.1039/C1CS15060J

[4] A. Hauch, A. Georg, U.O. Krasovec, B. Orel, J. Electrochem. Soc., 149, A1208-A1211 (2002). https://doi.org/10.1149/1.1500346

[5] F.R. Fan, Z.Q. Tian, Z.L. Wang, Nano Energy, 1, 328-334 (2012). https://doi.org/10.1016/j.nanoen.2012.01.004

[6] K. Zhao, Z.L. Wang, Y. Yang, ACS Nano, 10, 9044-9052 (2016). https://doi.org/10.1021/acsnano.6b05815

[7] Y. Tanaka, S. Funano, Y. Nishizawa, N. Kamamichi, M. Nishinaka, T. Kitamori, Sci. Rep., 6, 25899 (2016). https://doi.org/10.1038/srep25899

[8] K. Catania, Sci., 346, 1231-1234 (2014). https://doi.org/10.1126/science.1260807

[9] C. Wang, E. Choi, J. Park, Nano Energy, 43, 291-299 (2018). https://doi.org/10.1016/j.nanoen.2017.11.054

[10] M. Gottwald, N. Singh, A.N. Haubrich, S. Regett, G. Emde, Curr. Biol., 28, 3648-3653 (2018). https://doi.org/10.1016/j.cub.2018.09.036

[11] H. Izadi, K. M. E. Stewart, A. Penlidis, J. R. Soc. Interface., 11, 20140371 (2014). https://doi.org/10.1098/rsif.2014.0371

[12] T. B. H. Schroeder, A. Guha, A. Lamoureux, G. VanRenterghem, D. Sept, M. Shtein, J. Yang, M. Mayer, Nature, 552, 214-218 (2017). https://doi.org/10.1038/nature24670

[13] A. Chandrasekhar, G. Khandelwal, N.R. Alluri, V. Vivekananthan, S.-J. Kim, ACS Sustain. Chem. Eng., 6, 6110-6116 (2018). https://doi.org/10.1021/acssuschemeng.7b04769

[14] P. Maharjan, R.M. Toyabur, J.Y. Park, Nano Energy, 46, 383-395 (2018). https://doi.org/10.1016/j.nanoen.2018.02.033

[15] G. Zhu, C. Pan, W. Guo, C.-Y. Chen, Y. Zhou, R. Yu, Z. L. Wang, Nano Lett., 12, 4960-4965 (2012). https://doi.org/10.1021/nl302560k

[16] J.R. Gallant, L.L. Traeger, J.D. Volkening, H. Moffett, P.H. Chen, C.D. Novina, G.N. Phillips Jr., R. Anand, G.B. Wells, M. Pinch, R. Güth, G.A. Unguez, J.S. Albert, H.H. Zakon, M.P. Samanta, M.R. Sussman, Sci., 344, 1522-1525 (2014). https://doi.org/10.1126/science.1254432

[17] U. Khan, R. Hinchet, H. Ryu, S.W. Kim, APL Mater., 5, 073803 (2017). https://doi.org/10.1063/1.4979954

[18] S. Niu, Z.L. Wang, Nano Energy, 14, 161-192 (2015). https://doi.org/10.1016/j.nanoen.2014.11.034

[19] H. Yang, M. Deng, Q. Tang, W. He, C. Hu, Y. Xi, R. Liu, Z.L. Wang, Adv. Energy Mater., 9, 1901149 (2019). https://doi.org/10.1002/aenm.201901149

[20] X. Wen, Y. Su, Y. Yang, H. Zhang, Z.L. Wang, Nano Energy, 4, 2014, 150-156. https://doi.org/10.1016/j.nanoen.2014.01.001

[21] Y. Su, Y. Yang, X. Zhong, H. Zhang, Z. Wu, Y. Jiang, Z.L. Wang, ACS Appl. Mater. Interfaces, 6, 2014, 553-559. https://doi.org/10.1021/am404611h

[22] H. Wang, Z. Xiang, P. Giorgia, X. Mu, Y. Yang, Z.L. Wang, C. Lee, Nano Energy, 23, 2016, 80-88. https://doi.org/10.1016/j.nanoen.2016.02.054

[23] Y. Yang, H. Zhang, Z.L. Wang, Adv. Funct. Mater., 24, 2014, 3745-3750. https://doi.org/10.1002/adfm.201304295 
[24] X. Liu, K. Zhao, Z.L. Wang, Y. Yang, Adv. Energy Mater., 7, 2017, 1701629. https://doi.org/10.1002/aenm.201701629

[25] K. Zhao, Y. Yang, X. Liu, Z.L. Wang, Adv. Energy Mater., 7, 2017, 1700103. https://doi.org/10.1002/aenm.201700103

[26] B. Chen, N. Yang, Q. Jiang, W. Chen, Y. Yang, Nano Energy, 44, 2018, 468-475. https://doi.org/10.1016/j.nanoen.2017.12.026

[27] X. Zhao, D. Zhang, S. Xu, W. Qian, W. Han, Z.L. Wang, Y. Yang, Nano energy, 75, 2020, 104920. https://doi.org/10.1016/j.nanoen.2020.104920

[28] Q. Tang, X. Pu, Q. Zeng, H. Yang, J. Li, Y. Wu, H. Guo, Z. Huang, C. Hu, Nano Energy, 66, 104087 (2019). https://doi.org/10.1016/j.nanoen.2019.104087

[29] A. Chortos, Z. Bao, Mater. Today, 17, 321-331 (2014). https://doi.org/10.1016/j.mattod.2014.05.006

[30] H. Sun, X. Fu, S. Xie, Y. Jiang, H. Peng, Adv. Mater., 28, 2070-2076 (2016). https://doi.org/10.1002/adma.201505742

[31] Y. -C. Lai, J. Deng, S. Niu, W. Peng, C. Wu, R. Liu, Z. Wen, Z. L. Wang, Adv. Mater., 28, 10024-10032 (2016). https://doi.org/10.1002/adma.201603527

[32] J. Keesey, J. Hist. Neurosci., 14, 149-164 https://doi.org/10.1080/096470490512599

[33] Y. Jie, Q. Jiang, Y. Zhang, N. Wang, X. Cao, Nano Energy, 27, 554-560 (2016). https://doi.org/10.1016/j.nanoen.2016.07.028

[34] H.Y. Mi, X. Jing, Q. Zheng, L. Fang, H.X. Huang, L.S. Turng, S. Gong, Nano Energy, 48, 327-336 (2018). https://doi.org/10.1016/j.nanoen.2018.03.050

[35] J. Wang, Z. Wen, Y. Zi, P. Zhou, J. Lin, H. Guo, Y. Xu, Z. L. Wang, Adv. Funct. Mater., 26, 1070-1076 (2016). https://doi.org/10.1002/adfm.201504675

[36] Q. Zheng, L. Fang, H. Guo, K. Yang, Z. Cai, M. A. B. Meador, S. Gong, Adv. Funct. Mater., 28, 1706365 (2018). https://doi.org/10.1002/adfm.201706365

[37] H. Y.Mi, X. Jing, Z. Cai, Y. Liu, L. S. Turng, S. Gong, Nanoscale, 10, 23131-23140 (2018). https://doi.org/10.1039/C8NR05872E

[38] C. Zhang, X. Lin, N. Zhang, Y. Lu, Z. Wu, G. Liu, S. Nie, Nano Energy, 66, 104126 (2019). https://doi.org/10.1016/j.nanoen.2019.104126

[39] K. Zhang, P. Tao, Y. Zhang, X. Liao, S. Nie, Carbohydr. Polym., 213, 228-235 (2019). https://doi.org/10.1016/j.carbpol.2019.02.087

[40] C. Liu, J. Li, L. Che, S. Chen, Z. Wang, X. Zhou, Nano Energy, 41, 359-366 (2017). https://doi.org/10.1016/j.nanoen.2017.09.038

[41] F. Peng, D. Liu, W. Zhao, G. Zheng, Y. Ji, K. Dai, L. Mi, D. Zhang, C. Liu, C. Shen, Nano Energy, 65, 104068 (2019). https://doi.org/10.1016/j.nanoen.2019.104068

[42] W. Wang, A. Yu, X. Liu, Y. Liu, Y. Zhang, Y. Zhu, Y. Lei, M. Jia, J. Zhai, Z. L. Wang, Nano Energy, 71, 104605 (2020). https://doi.org/10.1016/j.nanoen.2020.104605

[43] R. Yuan, H. Li, X. Yin, P. Wang, J. Lu, L. Zhang, Chem. Commun., 55, 9031-9034 (2019). https://doi.org/10.1039/C9CC04470A

[44] J. Zhao, Z. Li, T. Shen, X. Yuan, G. Qiu, Q. Jiang, Y. Lin, G. Song, A. Meng, Q. Li, J. Mater. Chem. A, 7, 7918-7931 (2019). https://doi.org/10.1039/C8TA11953H

[45] V. Aravindan, J. Gnanaraj, Y. S. Lee, S. Madhavi, Chem. Rev., 114, 11619-11635 (2014). https://doi.org/10.1021/cr5000915

[46] M. Vangari, T. Pryor, L. Jiang, J. Energy Eng., 139, 72-79 (2013). https://doi.org/10.1061/(ASCE)EY.1943-7897.0000102

[47] Z. Niu, L. Liu, L. Zhang, W. Zhou, X. Chen, S. Xie, Adv Energy Mater., 5, 1500677 (2015). https://doi.org/10.1002/aenm.201500677 
[48] Z.-Q. Hao, J.-P. Cao, Y. Wu, X.-Y. Zhao, Q.-Q. Zhuang, X.-Y. Wang, X.-Y. Wei, J. Power Sources, 36, 249-258 (2017). https://doi.org/10.1016/j.jpowsour.2017.06.086

[49] H. Pan, J. Li, Y. Feng, Nanoscale Res. Lett., 5, 654-668 (2010). https://doi.org/10.1007/s11671-009-9508-2

[50] D.P. Dubal, S.H. Lee, J.G. Kim, W.B. Kim, C.D. Lokhande, J. Mater. Chem., 22, 30443052 (2012). https://doi.org/10.1039/C2JM14470K

[51] Q. Lu, J.G. Chen, J.Q. Xiao, Angew. Chem. Int. Ed., 52, 1882-1889 (2013). https://doi.org/10.1002/anie.201203201

[52] A. Muzaffar, M.B. Ahamed, K. Deshmukh, J. Thirumalai, Renew. Sustain. Energy Rev., 101, 123-145 (2019). https://doi.org/10.1016/j.rser.2018.10.026

[53] P.C. Chen, G. Shen, Y. Shi, H. Chen, C. Zhou, ACS Nano, 4, 4403-4411 (2010). https://doi.org/10.1021/nn100856y

[54] X. Chen, R. Paul, L. Dai, Natl. Sci. Rev., 4, 453-489 (2017). https://doi.org/10.1093/nsr/nwx009

[55] C. Zhao, W. Zheng, Front. Energy Res., 3, 1-11 (2015). https://doi.org/10.3389/fenrg.2015.00023

[56] R. B. Choudhary, S. Ansari, B. Purty, J. Energy Storage, 29, 101302 (2020). https://doi.org/10.1016/j.est.2020.101302

[57] C. Zhong, Y. Deng, W. Hu, J. Qiao, L. Zhang, J. Zhang, Chem. Soc. Rev., 44, 74847530 (2015). https://doi.org/10.1039/C5CS00303B

[58] X. Peng, L. Peng, C. Wu, Y.Xie, Chem. Soc. Rev., 43, 3303-3323 (2014). https://doi.org/10.1039/C3CS60407A

[59] J.B. Goodenough, Y. Kim, Chem. Mater., 22, 587-603 (2010). https://doi.org/10.1021/cm901452z

[60] Y. Cheng, H. Zhang, S. Hu, C. V. Varanasi, J. Liu, Nanoscale, 5, 1067-1073 (2013). https://doi.org/10.1039/C2NR33136E

[61] P. Yang, Y. Ding, Z. Lin, Z. Chen, Y. Li, P. Qiang, M. Ebrahimi, W. Mai, C.P. Wong, Z.L. Wang, Nano. Lett., 14, 731-736 (2014). https://doi.org/10.1021/nl404008e

[62] F. Grote, Y. Lein, Nano Energy, 10, 63-70 (2014). https://doi.org/10.1016/j.nanoen.2014.08.019

[63] Y. Chen, B. Liu, Q. Liu, J. Wang, J. Liu, H. Zhang, S. Hu, X. Jing, Electrochim. Acta, 178, 429-438 (2015). https://doi.org/10.1016/j.electacta.2015.08.040

[64] W. Qiu, Q. Zhou, H. Xiao, C. Zhou, W. He, Y. Li, X. Lu, J. Mater. Chem. A, 8, 87228730 (2020). https://doi.org/10.1039/D0TA01423K

[65] J. Zhu, S. Tang, J. Wu, X. Shi, B. Zhu, X. Meng, Adv. Energy Mater., 7, 1601234 (2017). https://doi.org/10.1002/aenm.201601234

[66] C.V.V.M. Gopi, A.E. Reddy, H.J. Kim, J. Mater. Chem. A, 6, 7439-7448 (2018). https://doi.org/10.1039/C8TA01141A

[67] Q. Liu, X. Hong, X. Zhang, W. Wang, W. Guo, X. Liu, M. Ye, Chem. Eng. J., 356, 985993 (2019). https://doi.org/10.1016/j.cej.2018.09.095

[68] G. Zhao, Y. Tang, G. Wan, X. Xu, X. Zhou, M. Zhou, C. Hao, S. Deng, G. Wang, J. Colloid Interface Sci., 572, 151-159 (2020). https://doi.org/10.1016/j.jcis.2020.03.084

[69] J. L. Liu, M. H. Chen, L. L. Zhang, J. Jiang, J. X. Yan, Y. Z. Huang, J. Y. Lin, H. J. Fan, Z. X. Shen, Nano Lett., 14, 7180-7187 (2014). https://doi.org/10.1021/n1503852m

[70] H. Tan, Z. H. Liu, D. L. Chao, P. Hao, D. D. Jia, Y. H. Sang, H. Liu, H. J. Fan, Adv. Energy Mater., 8, 1800685 (2018). https://doi.org/10.1002/aenm.201800685

[71] S. Wang, Z.-H. Lin, S. Niu, L. Lin, Y. Xie, K.C. Pradel, Z.L. Wang, ACS Nano, 7, 11263-11271 (2013). https://doi.org/10.1021/nn4050408

[72] P.K. Yang, Z.H. Lin, K.C. Pradel, L.Lin, X. Li, X. Wen, J.H. He, Z.L. Wang, ACS Nano, 9, 901-907 (2015). https://doi.org/10.1021/nn506631t 
[73] P.K. Yang, L. Lin, F. Yi, X. Li, K.C. Pradel, Y. Zi, C.I. Wu, J.H. He, Y. Zhang, Z.L. Wang, Adv. Mater. 27, 3817-3824 (2015). https://doi.org/10.1002/adma.201500652

[74] N. Sun, Z. Wen, F. Zhao, Y. Yang, H. Shao, C. Zhou, Q. Shen, K. Feng, M. Peng, Y. Li, X. Sun, Nano Energy, 38, 210-217 (2017). https://doi.org/10.1016/j.nanoen.2017.05.048

[75] H. Guo, M.-H. Yeh, Y. Zi, Z. Wen, J. Chen, G. Liu, C. Hu, Z. L. Wang, ACS Nano, 11, 4475-4482 (2017). https://doi.org/10.1021/acsnano.7b00866

[76] J. Wang, X. Li, Y. Zi, S. Wang, Z. Li, L. Zheng, F. Yi, S. Li, Z.L. Wang, Adv. Mater., 27, 4830-4836 (2015). https://doi.org/10.1002/adma.201501934

[77] X. Pu, L. Li, M. Liu, C. Jiang, C. Du, Z. Zhao, W. Hu, Z. L. Wang, Adv. Mater., 28, 98105 (2016). https://doi.org/10.1002/adma.201504403

[78] J. Zhao, H. Li, C. Li, Q. Zhang, J. Sun, X. Wang, J. Guo, L. Xie, J. Xie, B. He, Z. Zhou, C. Lu, W. Lu, G. Zhu, Y. Yao, Nano Energy, 45, 420-431 (2018). https://doi.org/10.1016/j.nanoen.2018.01.021

[79] W. Xiong, K. Hu, Z. Li, Y. Jiang, Z. Li, Z. Li, X. Wang, Nano Energy, 66, 104149 (2019). https://doi.org/10.1016/j.nanoen.2019.104149

[80] J. Chen, H. Guo, X. Pu, X. Wang, Y. Xi, C. Hu, Nano Energy, 50, 536-543 (2018). https://doi.org/10.1016/j.nanoen.2018.06.009

[81] K. Dong, Y.C. Wang, J. Deng, Y. Dai, S.L. Zhang, H. Zou, B. Gu, B. Sun, Z.L. Wang, ACS Nano, 11, 9490-9499 (2017). https://doi.org/10.1021/acsnano.7b05317

[82] M. Liu, Z. Cong, X. Pu, W. Guo, T. Liu, M. Li, Y. Zhang, W. Hu, Z. L. Wang, Adv. Funct. Mater., 29, 1806298 (2019). https://doi.org/10.1002/adfm.201806298

[83] S. Li, S. Wang, Y. Zi, Z. Wen, L. Lin, G. Zhang, Z.L. Wang, ACS Nano, 9, 7479-7487 (2015). https://doi.org/10.1021/acsnano.5b02575

[84] J. Wang, Y. Xu, X. Chen, X. Du, J. Power Sources, 163, 1120-1125 (2007). https://doi.org/10.1016/j.jpowsour.2006.10.004

[85] Y. Song, X. Cheng, H. Chen, J. Huang, X. Chen, M. Han, Z. Su, B. Meng, Z. Song, H. Zhang, J. Mater. Chem. A, 4, 14298-14306 (2016). https://doi.org/10.1039/C6TA05816G

[86] B. K. Deka, A. Hazarika, S. Lee, D. Y. Kim, Y. B. Park, H. W. Park, Nano Energy, 73, 104754 (2020). https://doi.org/10.1016/j.nanoen.2020.104754

[87] F. Yi, J. Wang, X. Wang, S. Niu, S. Li, Q. Liao, Y. Xu, Z. You, Y. Zhang, Z.L. Wang, ACS Nano, 10, 6519-6525 (2016). https://doi.org/10.1021/acsnano.6b03007

[88] H. Guo, M.-H. Yeh, Y.-C. Lai, Y. Zi, C. Wu, Z. Wen, C Hu, Z.L. Wang, ACS Nano, 10, 10580-10588 (2016). https://doi.org/10.1021/acsnano.6b06621

[89] D. Pech, M. Brunet, H. Durou, P. Huang, V. Mochalin, Y. Gogotsi, P. Taberna, P. Simon, Nat. Nanotechnol., 5, 651-654 (2010). https://doi.org/10.1038/nnano.2010.162

[80] J. Luo, F.R. Fan, T. Jiang, Z. Wang, W. Tang, C. Zhang, M. Liu, G. Cao, Z.L. Wang, Nano Res., 8, 3934-3943 (2015). https://doi.org/10.1007/s12274-015-0894-8

[91] X. Wang, Y. Yin, F. Yi, K. Dai, S. Niu, Y. Han, Y. Zhang, Z. You, Nano Energy, 39, 429-436 (2017). https://doi.org/10.1016/j.nanoen.2017.07.022

[92] Q. Jiang, C. Wu, Z. Wang, A.C. Wang, J.-H. He, Z.L. Wang, H.N. Alshareef, Nano Energy, 45, 266-272 (2018). https://doi.org/10.1016/j.nanoen.2018.01.004

[93] Z. Wen, M.H. Yeh, H. Guo, J. Wang, Y. Zi, W. Xu, J. Deng, L. Zhu, X. Wang, C. Hu, L. Zhu, X. Sun, Z.L. Wang, Sci. Adv., 2, e1600097 (2016). https://doi.org/10.1126/sciadv.1600097

[94] W. S. Jung, M. G. Kang, H. G. Moon, S. H. Baek, S. J. Yoon, Z. L. Wang, S. W. Kim, C. Y. Kang, Sci. Rep., 5, 9309 (2015). https://doi.org/10.1038/srep09309

[95] S. Qin, Q. Zhang, X. Yang, M. Liu, Q. Sun, Z.L. Wang, Adv. Energy Mater., 8, 1800069 (2018). https://doi.org/10.1002/aenm.201800069

[96] F.R. Fan, W. Tang, Y. Yao, J. Luo, C. Zhang, Z.L. Wang, Nanotechnology, 25, 135402 (2014). https://doi.org/10.1088/0957-4484/25/13/135402 
[97] G. Zhu, J. Chen, T. Zhang, Q. Jing, Z.L Wang, Nat Commun., 5, 3426 (2014). https://doi.org/10.1038/ncomms4426

[98] D. Bhatia, J. Lee, H.J. Hwang, J.M. Baik, S. Kim, D. Choi, Adv. Energy Mater., 8, 1702667 (2018). https://doi.org/10.1002/aenm.201702667

[99] C. Han, C. Zhang, W. Tang, X. Li, Z.L. Wang, Nano Res., 8, 722-730 (2015). https://doi.org/10.1007/s12274-014-0555-3

[100] Y. Zi, H. Guo, Z. Wen, M.H. Yeh, C. Hu, Z.L. Wang, ACS Nano, 10, 4797-4805 (2016). https://doi.org/10.1021/acsnano.6b01569

[101] S. Niu, X. Wang, F. Yi, Y.S. Zhou, Z.L. Wang, Nat. Commun., 6, 8975 (2015). https://doi.org/10.1038/ncomms9975

[102] X. Cheng, L. Miao, Y. Song, Z. Su, H. Chen, X. Chen, J. Zhang, H. Zhang, Nano Energy, 38, 438-446 (2017). https://doi.org/10.1016/j.nanoen.2017.05.063

[103] F. Xi, Y. Pang, W. Lia, T. Jiang, L. Zhang, T. Guo, G. Liu, C. Zhang, Z.L. Wang, Nano Energy, 37, 168-176 (2017). https://doi.org/10.1016/j.nanoen.2017.05.027

[104] X. Liang, T. Jiang, G. Liu, T. Xiao, L. Xu, W. Li, F. Xi, C. Zhang, Z.L. Wang, Adv. Funct. Mater., 29, 1807241 (2019). https://doi.org/10.1002/adfm.201807241

[105] W. Tang, T. Zhou, C. Zhang, F.R. Fan, C.B. Han, Z.L. Wang, Nanotechnology, 25, 225402 (2014). https://doi.org/10.1088/0957-4484/25/22/225402

[106] Y. Zi, H. Guo, J. Wang, Z. Wen, S. Li, C. Hu, Z.L. Wang, Nano Energy, 31, 302-310 (2017). https://doi.org/10.1016/j.nanoen.2016.11.025

[107] Y. Zi, J. Wang, S. Wang, S. Li, Z. Wen, H. Guo, Z.L. Wang, Nat. Commun., 7, 10987 (2016). https://doi.org/10.1038/ncomms10987 